\title{
Antagonism between abscisic acid and ethylene in Arabidopsis acts in parallel with the reciprocal regulation of their metabolism and signaling pathways
}

\author{
Wan-Hsing Cheng $\cdot$ Ming-Hau Chiang • \\ San-Gwang Hwang • Pei-Chi Lin
}

Received: 10 March 2009/Accepted: 24 May 2009/Published online: 10 June 2009

(c) The Author(s) 2009. This article is published with open access at Springerlink.com

\begin{abstract}
Although abscisic acid (ABA) and ethylene have antagonistic functions in the control of plant growth and development, including seed germination and early seedling development, it remains unknown whether a convergent point exists between these two signaling pathways or whether they operate in parallel in Arabidopsis thaliana. To elucidate this issue, four ethylene mutants, ctr1, ein2, ein3, and ein6, were crossed with aba2 (also known as gin1-3) to generate double mutants. Genetic epistasis analysis revealed that all of the resulting double mutants displayed aba2 mutant phenotypes with a small plant size and wiltiness when grown in soil or on agar plates. Further ethylene sensitivity or triple response analyses demonstrated that these double mutants also retained the ctrl or ein mutant phenotypes, showing ethylene constitutive triple and insensitive responses, respectively. Our current data therefore demonstrate that ABA and ethylene act in parallel, at least in primary signal transduction pathways. Moreover, by microarray analysis we found that an ACC oxidase (ACO) was significantly upregulated in the aba2 mutant, whereas the 9-CIS-EPOXYCAROTENOID DIOXYGENASE 3 (NCED3) gene in ein2 was upregulated, and both the ABSCISIC ACID INSENSITIVE1 (ABII) and cytochrome P450, family 707, subfamily A, polypeptide 2 (CYP707A2) genes in etr1-1 were downregulated. These data further suggest that ABA and ethylene may control the hormonal biosynthesis,
\end{abstract}

Electronic supplementary material The online version of this article (doi:10.1007/s11103-009-9509-7) contains supplementary material, which is available to authorized users.

W.-H. Cheng $(\bowtie) \cdot$ M.-H. Chiang · S.-G. Hwang · P.-C. Lin Institute of Plant and Microbial Biology, Academia Sinica, Taipei, Taiwan, Republic of China

e-mail: whcheng@gate.sinica.edu.tw catabolism, or signaling of each other to enhance their antagonistic effects upon seed germination and early seedling growth.

Keywords Abscisic acid - Ethylene - Antagonism . ABA2 - EIN2

$\begin{array}{ll}\text { Abbreviations } \\ \text { ABA } & \text { Abscisic acid } \\ \text { ABI } & \text { Abscisic acid insensitive } \\ \text { ACO } & \text { ACC oxidase } \\ \text { CPK } & \text { Calcium-dependent protein kinase } \\ \text { CYP707A2 } & \text { Cytochrome P450, family 707, subfamily A, } \\ & \text { polypeptide 2 } \\ \text { ERA1 } & \text { Enhanced response to ABA1 } \\ \text { ERF } & \text { Ethylene response factor } \\ \text { gin } & \text { Glucose insensitive } \\ \text { glo } & \text { Glucose oversensitive } \\ \text { Lox2 } & \text { Lipoxygenase } \\ \text { NCED } & \text { 9-cis-epoxycarotenoid dioxygenase } \\ \text { SnRK } & \text { SNF1-related protein kinase } \\ \text { VSP1 } & \text { Vegetative storage protein 1 } \\ \text { THI2.1 } & \text { Thionin }\end{array}$

\section{Introduction}

Abscisic acid (ABA) is a classic phytohormone that plays an important role in various aspects of plant growth and development, including seed maturation and dormancy (Karssen et al. 1983; Koornneef et al. 1989), stomatal closure (Leung and Giraudat 1998), and adaptation to environmental stress (Qin and Zeevaart 2002; Xiong et al. 2002). In the past decade, significant advances have been made to better understand the expression and regulation of 
ABA biosynthesis and its signaling genes at the molecular level. Quite recently, most of the key components involved in ABA biosynthesis have been isolated and characterized through genetic and biochemical screens for ABA-deficient mutants in Arabidopsis that show an early germination phenotype (North et al. 2007; Dall'Osto et al. 2007; for reviews, see Finkelstein et al. 2002; Seo and Koshiba 2002; Schwartz et al. 2003; Xiong and Zhu 2003). For instance, ABA1, a zeaxanthin epoxidase (ZEP), catalyzes the epoxidation of zeaxanthin and antheraxanthin to violaxanthin in plastids (Marin et al. 1996; Xiong et al. 2002). After structural modification, violaxanthin is converted to 9-cis-epoxycarotenoid through ABA4 activity and/or other isomerase(s) (North et al. 2007). The epoxycarotenoids 9'-cis-neoxanthin and/or 9-cis-violaxanthin are then oxidized by 9-cis-epoxycarotenoid dioxygenase (NCED) to generate a C15 intermediate, xanthoxin (Schwartz et al. 1997). The product xanthoxin is then transported to the cytosol and further converted to abscisic aldehyde by a short-chain dehydrogenase/reductase 1 , encoded by $A B A 2$ in Arabidopsis (Rook et al. 2001; Cheng et al. 2002; González-Guzmán et al. 2002). In the last step of ABA biosynthesis, abscisic aldehyde is oxidized to form abscisic acid by Arabidopsis aldehyde oxidase 3 (AAO3) (Seo et al. 2000), which needs a molybdenum cofactor sulfurase encoded by ABA3 (Bittner et al. 2001; Xiong et al. 2001) for its activity. Of these genes, ABA2 acts as a link between sugar and ABA signaling (Cheng et al. 2002) and its expression is upregulated by prolonged stress. Thus, it is proposed that $\mathrm{ABA} 2$ has a fine-tuning function in mediating $\mathrm{ABA}$ biosynthesis through primary metabolic changes in response to stress (Lin et al. 2007).

Likewise, genetic screens for reduced ABA inhibition of seed germination have identified several components that participate in $\mathrm{ABA}$ signaling including $\mathrm{ABI} 1$ to $\mathrm{ABI} 5$ and ABI8. $A B I 1$ and $A B I 2$ encode homologous serine/threonine phosphatase $2 \mathrm{C}$ proteins (Leung et al. 1997) that play a negative role in ABA signal transduction (Sheen 1998; Gosti et al. 1999). ABI3 is an ortholog of maize VP1, a B3domain containing transcription factor (Brady et al. 2003). The ABI4 and ABI5 proteins belong to an APETALA2domain and a $\beta$-basic leucine zipper transcription factors, respectively (Finkelstein et al. 1998; Finkelstein and Lynch 2000). ABI8 is a novel protein of unknown function (Brocard-Gifford et al. 2004). In addition to genetic screens for mutants with reduced ABA sensitivity, ABA hypersensitive mutants have also been isolated and characterized. Most of the corresponding genes in these cases are involved in RNA processing and protein metabolism (for review, see Finkelstein et al. 2008). For instance, Enhanced Response to ABAl (ERAl), encoding a farnesyl transferase, is involved in the farnesylation of signaling proteins (Cutler et al. 1996). Mutation of RPN10, a subunit of the
26S proteosome, also causes ABA hypersensitivity (Smalle et al. 2003). However, the mechanisms by which RNA processing and protein metabolism alter ABA sensitivity in response to stress remain largely unknown. Recently, two SNF1-related protein kinases (SnRK2.2 and SnRK2.3) (Fujii et al. 2007) and two calcium-dependent protein kinases (CPK4 and CPK11) (Zhu et al. 2007) in Arabidopsis have been shown to regulate ABA signal transduction and to affect seed germination, root or seedling growth, and other phenotypes. These data further support the involvement of protein kinases in the ABA signaling effects that subsequently regulate plant growth and development.

In addition to ABA, ethylene is another stress-induced hormone with fundamental roles in germination, sex determination, leaf abscission, flower senescence, fruit ripening, and responses to biotic and abiotic stress (for review, see Johnson and Ecker 1998). It has been shown that a subset of the functions of ethylene overlaps with those of ABA. Ethylene, for instance, also participates in seed germination and in early seedling establishment, albeit with opposite effects to ABA (Zhou et al. 1998). The enhanced response to $A B A 3$ (era3) mutant was shown to be a new allele of ein2 that shows hypersensitivity to $\mathrm{ABA}$ in seed germination, but an insensitivity to $\mathrm{ABA}$ in root growth (Ghassemian et al. 2000). Similarly, ctrl and ein2 were recovered as an enhancer and a suppressor, respectively, of the ABA-resistant seed germination of abil-1 (Beaudoin et al. 2000). CTR1 belongs to the Raf family of Ser/Thr protein kinases and negatively regulates ethylene signaling (Kieber et al. 1993). The mutation of CTR1 in the ctr1-1 mutant causes an ethylene constitutive triple response and insensitivity to sugar (Zhou et al. 1998). EIN2 is a central component of ethylene signaling and plays important roles in crosslinking multiple hormones and stress (Alonso et al. 1999; Wang et al. 2007). It was also reported that ABA-deficient mutants of Arabidopsis aba2 and tomato flacca and notabilis reveal inhibition of shoot growth, largely because of high ethylene production in these mutants (Sharp et al. 2000; LeNoble et al. 2004). Hence, the ABA and ethylene signaling pathways have a close interplay in plant growth, development, and stress response. However, it remains unknown whether their respective signal transduction pathways have any convergent points or function only in parallel. To elucidate this issue, four double mutants were generated by crossing the ethylene mutants, $c t r 1$, ein 2 , ein 3 , and ein6, with the aba2 (or gin1-3) mutant. The reason that we used $a b a 2$ in this study rather than an ABA signal mutant is that the ABA has multiple sites of perception and signaling pathways. All $A B I$ genes identified to date only respond to parts, but not all, of ABA or stress signal transduction pathways. However, the use of $a b a 2$ lacking ABA biosynthesis will thus block all of ABA signal transduction pathways. Genetic 
epistasis analysis revealed that these double mutants displayed both an aba2 and a ctrl/or ein mutant phenotypes, i.e., a small plant size and either a constitutive triple response or insensitivity to ethylene. This indicates that ABA and ethylene act in parallel, at least during primary (or early) signal transduction. Further microarray analysis of the aba2, ein2, and etrl mutants suggested that ABA and ethylene may control the hormonal biosynthesis, catabolism, or signaling of each other to enhance their mutually antagonistic effects upon seed germination and early seedling growth.

\section{Materials and methods}

Plant materials and growth conditions

Plant materials used in this study were the Arabidopsis (Arabidopsis thaliana) ecotypes Columbia (Col) and Lanberg errata (Ler). The Col ecotype was used in most experiments. The aba2 mutant is the glucose insensitive 1-3 (gin1-3) allele with a 53-bp deletion at the start of exon 2 (Cheng et al. 2002). The ethylene mutants included in this study were etrl-1 (Col), ctrl-1 (Col), ein2-1 (Col), ein3-1 (Col), and ein6-1 (Ler), all of which harbor mutations in genes involved in the ethylene signal transduction pathway; the corresponding mutant seeds were requested from the Arabidopsis Biological Resource Center (ABRC). The etol-4 (Col) mutant, which causes ethylene overproduction, was also used in this study. All seeds were sterilized and subjected to cold pretreatment at $4{ }^{\circ} \mathrm{C}$ for 3 days in the dark, and then grown on agar plates or in soil at the first day of germination or planting. Seed germination and seedling growth were performed at $24^{\circ} \mathrm{C}$ under a long day (16-h light/8-h dark cycle) with a light intensity of $\sim 80 \mu \mathrm{Es}^{-1} \mathrm{~m}^{-2}$. Details of the seed sterility and medium preparation methods have been described previously (Lin et al. 2007).

Isolation of the ctrlaba2 or einaba2 double mutants

For the double mutant isolation, the ctrl and ein mutants (ein2, ein3, and ein6) were crossed with the aba2 mutant to generate F1 seeds. These F1 seeds were then germinated in soil, and mature plants were self pollinated to produce F2 seeds. Subsequently, the F2 seeds were grown on $1 \%$ sucrose medium supplemented with or without $10 \mu \mathrm{M}$ ACC for 5 days in the dark. For the ctrlaba2 double mutant, the $\mathrm{F} 2$ etiolated seedlings grown on $1 \%$ sucrose medium without ACC and showing an ethylene triple response were transferred to the same fresh medium under light conditions for 14 days prior to transplantation to soil. For the einaba2 double mutants, etiolated seedlings grown on medium with $10 \mu \mathrm{M}$ ACC and showing an ethyleneinsensitive phenotype were transferred to the same fresh medium without ACC under light conditions for 14 days prior to transplanting to soil. The genotypes of the double mutants were further confirmed by genomic PCR using primers that amplified the 53-bp deletion region in exon 2 of the aba 2 allele as described previously (Lin et al. 2007). This testing was performed for at least one further generation to validate the double mutants with an ethyleneresponsive phenotype and an aba2 background.

Isolation of the aco and acoaba2 double mutants

The aco mutant seeds for a SALK T-DNA line (Alonso et al. 2003) were requested from the ABRC stock center (Columbus, OH) with the accession no. SALK_082132. The T-DNA insertion at the ACO (At1g12010) locus was confirmed by genotyping. RT-PCR analysis confirmed that the aco mutant is a null mutant with an undetectable transcript. For the acoaba 2 double mutant isolation, the aco plant was crossed with $a b a 2$. In the resulting F2 segregating population, the acoaba2 double mutant was identified by genotyping for the respective mutant alleles. The putative double mutant was thus confirmed and showed no segregation in the F3 generation.

\section{Germination and root elongation tests}

For germination testing, seeds were sterilized, cold pretreated, and then grown on medium supplemented with sucrose or glucose. The medium was autoclaved and cooled to $50-60^{\circ} \mathrm{C}$ prior to the addition of filter-sterilized ABA [( \pm$)$-abscisic acid; Sigma; catalog no. A-1049], ACC (1-aminocyclopropane-carboxylic acid; Sigma; catalog no. A3903), or AVG [L- $\alpha$-(2-aminoethxyvinyl)glycine hydrochloride; Sigma; catalog no. A6685] at the various concentrations indicated in the text. The procedure for the root elongation analysis has been described previously (Lin et al. 2007).

Dehydration and water loss experiments

For dehydration, seeds subjected to cold pretreatment were grown in soil for 21 days, and subsequently pots were soaked in water for $5 \mathrm{~min}$. The excess water was removed by transferring pots to a clean tray for another $5 \mathrm{~min}$, and the plants were transferred to a new clean tray where watering was withheld for the periods indicated in the text. For water loss tests, the soil-grown plants at 21 days of age were excised and the aerial parts of tissues were placed on plastic weigh boats and kept in an electronic dry box (model-DX-76, Taiwan-DX-76, Taiwan Dry Tech Corp.) with a relative humidity of approximately $40 \%$. The fresh 
weights of the tissues were measured at 30-min intervals for $3 \mathrm{~h}$.

Ethylene and ABA measurements

To assay for ethylene, the sterilized seeds having undergone a cold pretreatment were sown in 20-ml flat-bottom Headspace vials (catalog no. 5182-0837, Agilent Technologies, Santa Clara, CA), each with 33 seeds. Each vial containing $10 \mathrm{ml}$ of modified MS medium supplemented with $1 \%$ sucrose was sealed using a crimp cap (catalog no. 5183-4477, Agilent Technologies) and placed in a culture room at $24^{\circ} \mathrm{C}$ in the dark for 3 or 4 days. Subsequently, the vials containing etiolated seedlings were arranged in a Headspace Sampler (Aglient G1888 Network Headspace Sampler) with a connection to gas chromatography (Hewlett Packard HP6890 GC System) to measure the ethylene contents. The measurement protocols essentially followed the manufacturer's instructions.

For the ABA assay, seedlings grown on $1 \%$ sucrose agar plates for 12-14 days were harvested, followed by ABA extraction, purification, and measurement of $\mathrm{ABA}$ as described previously (Lin et al. 2007).

\section{Microarray assay}

Cold pretreated seeds were grown on $1 \%$ sucrose agar plates for 12-14 days. Total RNAs were extracted as described (Lin et al. 2007), and $10 \mu \mathrm{g}$ aliquots were then used for cDNA synthesis and labeling by in vitro transcription followed by fragmentation according to the GeneChip Expression Analysis Technical Manual rev5, Affymetrix. Each labeled sample $(11 \mu \mathrm{g})$ was hybridized to an ATH1 GeneChip at $45^{\circ} \mathrm{C}$ for $16.5 \mathrm{~h}$. The washing and staining steps were performed using a Fluidic Station-450, and the ATH1 slides were scanned using the Affymetrix GeneChip Scanner 7G. Subsequent data processing and analysis were performed using the Affymetrix Microarray Suit, version 5.0 software. Two independent sets of microarray analyses were performed in this study.

\section{Semiquantitative RT-PCR}

Seedlings grown on 1\% sucrose agar plates for 12-14 days were harvested and used for total RNA extraction. The extraction procedure and reverse transcriptase (RT)-PCR were followed the protocol described previously (Lin et al. 2007). For semiquantitative RT-PCR, at least three different program cycles were used to obtain the optimal PCR conditions. The resulting PCR products were separated and visualized on $1 \%$ agarose gels stained with ethidium bromide. The band intensity of RT-PCR products was quantified with Quantity One 4.5 version software (BioRad Laboratories).

\section{Results}

Phenotypic comparisons of single and double mutants for the ABA and ethylene signaling pathways

Although ABA and ethylene function antagonistically in several aspects of plant growth and development, including seed germination and postgermination seedling establishment, it remains unknown whether there is any point of convergence between these two signaling pathways, or if they act in parallel only. To address this, four ethylene mutants, ctr1, ein2, ein3, and ein6, were crossed with the aba2 (or gin1-3) mutant to generate double mutants. As ctrl (constitutive triple response1) is a loss-of-function mutant that manifests as a negative regulator of CTR1 in response to ethylene, the double mutant ctrlaba2 displayed a $c t r 1$ phenotype with small leaves and plant size (Kieber et al. 1993) when grown on $1 \%$ sucrose agar plates for 14 days (Fig. 1A) or in soil for 21 or 42 days (Fig. 1B, C). Our detailed analysis further showed that the ctrlaba2 double mutant developed a wilty phenotype (data not shown) and a smaller plant size than that of the ctrl single mutant (Fig. 1). Significantly, these are phenotypes that are typical of aba2. Similarly, combining a loss-of-function of the ein2, ein3, and ein6 alleles with the aba2 locus still produced a typical aba 2 appearance in the aerial portions of the plant, i.e., a small plant size and dark-green leaves, when grown on $1 \%$ sucrose agar plates or in soil (Fig. 1). Our previous data showed that the aba2 mutant has smaller siliques and fewer seeds than those of the wild type (Cheng et al. 2002). Similarly, in our current analysis, the double mutants also displayed smaller siliques and produced less seeds, compared with the corresponding ctrl or ein single mutants (data not shown). Taken together, these data suggest that the double mutants, ein2aba2 and ein3aba2, have phenotypes that closely resemble the aba2 mutant, except that the ctrlaba2 displays both the ctrl and aba2 phenotype, because $c t r l$ is a loss-of-function mutant and confers a constitutive ethylene signal transduction.

Comparison of the effects of drought treatment and water loss upon the single and double ABA/ ethylene mutants

In addition to a small plant size, the ABA-deficient mutant aba2 is characterized by a wilty phenotype. To confirm whether or not the double mutants also harbored this phenotype, 28-day-old plants were subjected to drought conditions. The results demonstrated that the 
Fig. 1 Phenotypic comparison of single and double ABA/ ethylene mutants. A Seeds were grown on $1 \%$ sucrose agar plates for 14 days. B Seeds were grown in soil for 21 days. C Seeds were grown in soil for 42 days. Three independent experiments were performed, each with at least 12 plants and giving consistent results. WT wild type
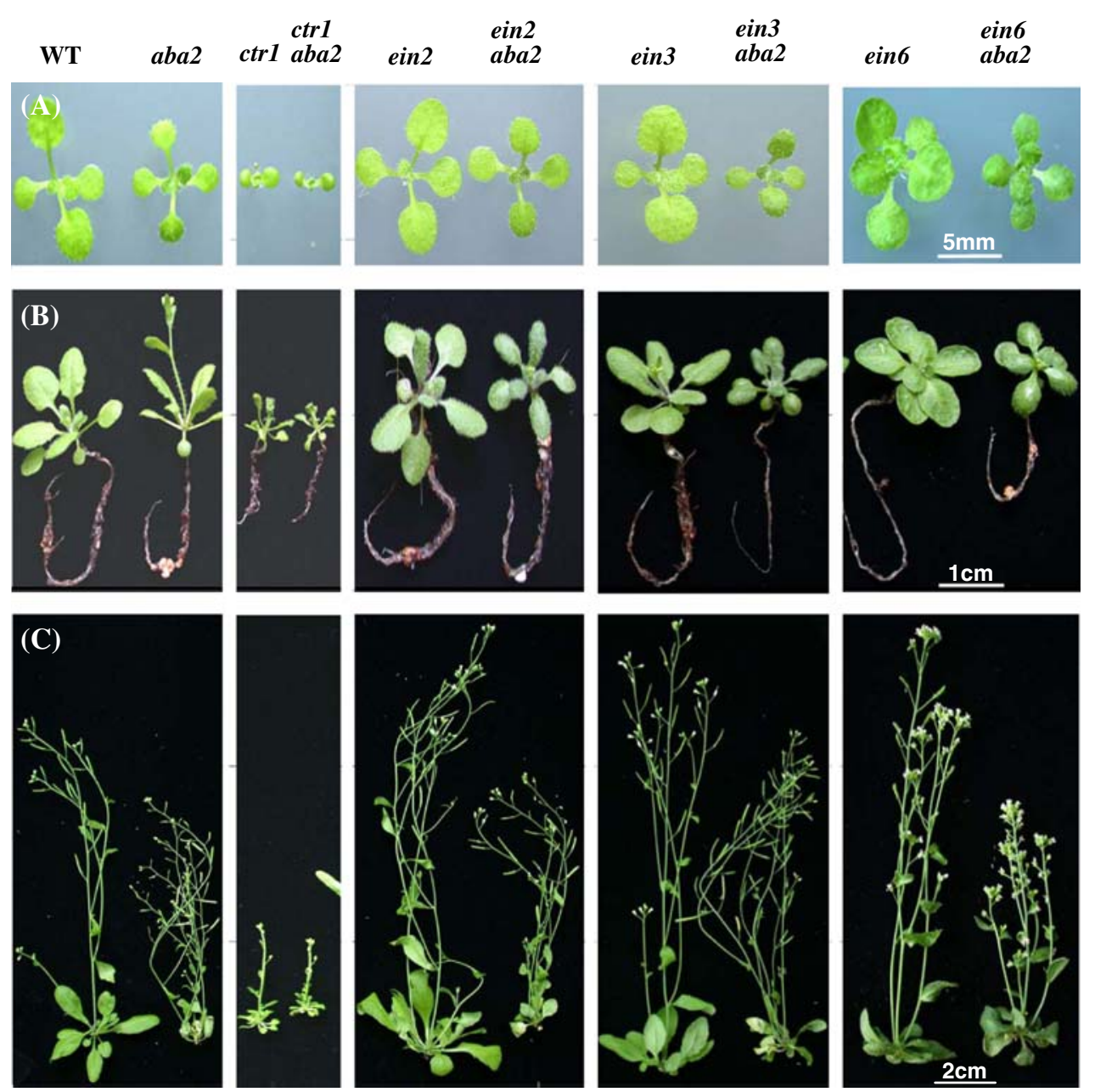

$a b a 2$, ein2aba2, and ein3aba2 mutants developed a severe wilty phenotype, after the withholding of water for 4 days (Fig. 2A). In contrast, the wild type, ein2, and ein3 plants retained a normal appearance even after 8 days of drought treatment. For relative water loss, the 28-day-old plants were removed, and the aerial regions were subjected to a water loss test. As shown in Fig. $2 \mathrm{~B}$ and $\mathrm{C}$, the ein2 and ein3 plants displayed a relative water loss pattern similar to the wild type, whereas the ein2aba2 and ein3aba2 double mutants showed a water loss pattern that resembled the aba2 single mutant. Similar results were also observed for ctrl versus ctrlaba2 and ein6 versus ein6aba2 comparisons (data not shown). Taken together, we conclude from these results that $a b a 2$ has greater effects on aerial phenotypes (wiltiness and plant size) than ein mutants do.

Comparison of the ethylene responses

of the ABA/ethylene mutants

Based on the aforementioned data, the ABA-signaling pathway has greater effects on aerial phenotypes than ethylene signaling does. As ethylene may induce root hair formation, we tested whether a constitutive triple ethylene response was gained in ctrlaba2 and whether insensitivity to the ethylene phenotype arose in the einaba2 mutants. All mutants were grown on $1 \%$ sucrose agar plates with or without ACC $(10 \mu \mathrm{M})$. As shown in Fig. 3, in the absence of ACC, only the ctrl and ctrlaba2 plants exhibited hairy roots, whereas the other genotypes showed no apparent induction of root hair formation (Fig. 3A). In the presence of ACC, in addition to $c t r 1$ and ctrlaba2, the wild-type and $a b a 2$ plants also displayed hairy roots at the newly growing roots, whereas the ein and einaba 2 mutants showed no apparent root hair formation. It is notable also that all of the genotypes tested in these analyses revealed short roots following treatment with ACC and that ctrlaba2 had more pronounced hairy root formation compared with its parental mutant ctrl (Fig. 3B). However, the mechanisms underlying the enhanced hairy root formation in ctrlaba2 remain unknown. In addition to above data, aba2 and each of the double mutants displayed a shorter primary root length on average than the wild-type and corresponding single mutant plants (Fig. 3A). 
Fig. 2 Comparison of the effects of drought and water loss upon single and double $\mathrm{ABA} /$ ethylene mutants. A Drought treatment. Seeds were grown in soil for 28 days, and the resulting plants did not receive water for 4 additional days. Two independent experiments were performed, each with plants $(n \geq 9)$, and gave consistent results. $\mathbf{B}$ and $\mathbf{C}$ Water loss test. Seeds were grown in soil for 28 days, and then the aerial portions were removed and subjected to a water loss test in an electronic dryer. The results shown are the means \pm SD of triplicate analyses in one experiment, and each repeat was with two to five plants. Two independent experiments were performed and gave consistent results

Fig. 3 Ethylene induction of root hair formation. Seeds were grown on $1 \%$ sucrose agar plates for 4 days, and the seedlings were transferred to the same fresh medium without ACC (A) or with $10 \mu \mathrm{M}$ ACC (B) and grown vertically for another 5 days. Three independent experiments were performed, giving consistent results; each experiment had seedlings $(n \geq 6)$. Scale bars represent $6 \mathrm{~mm}$
(A)
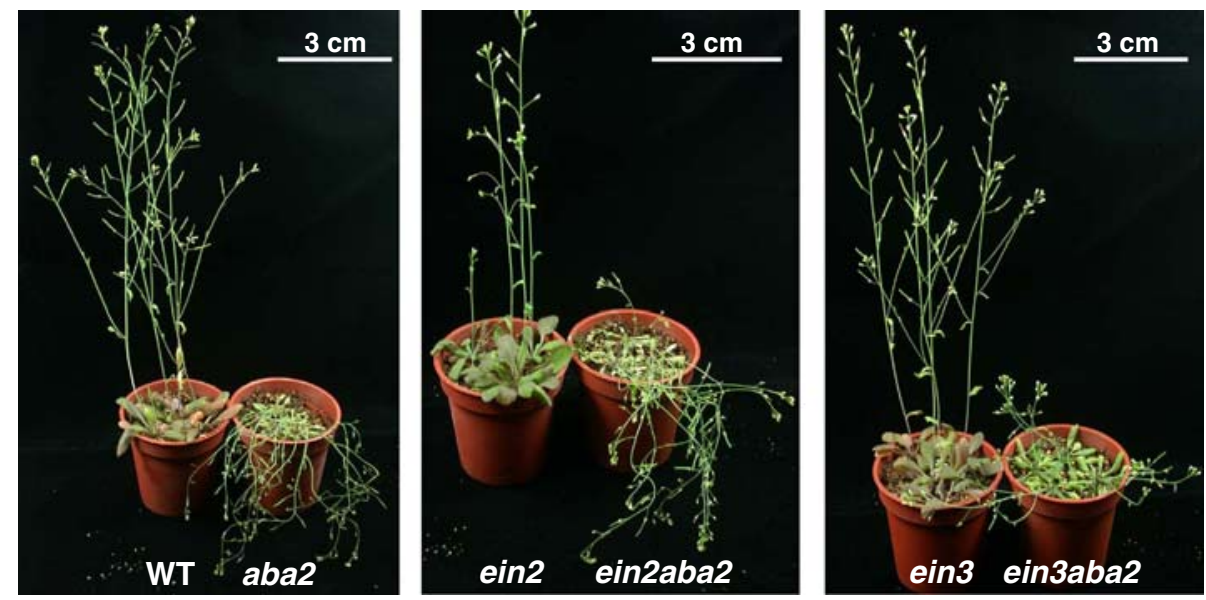

(B)

(C)
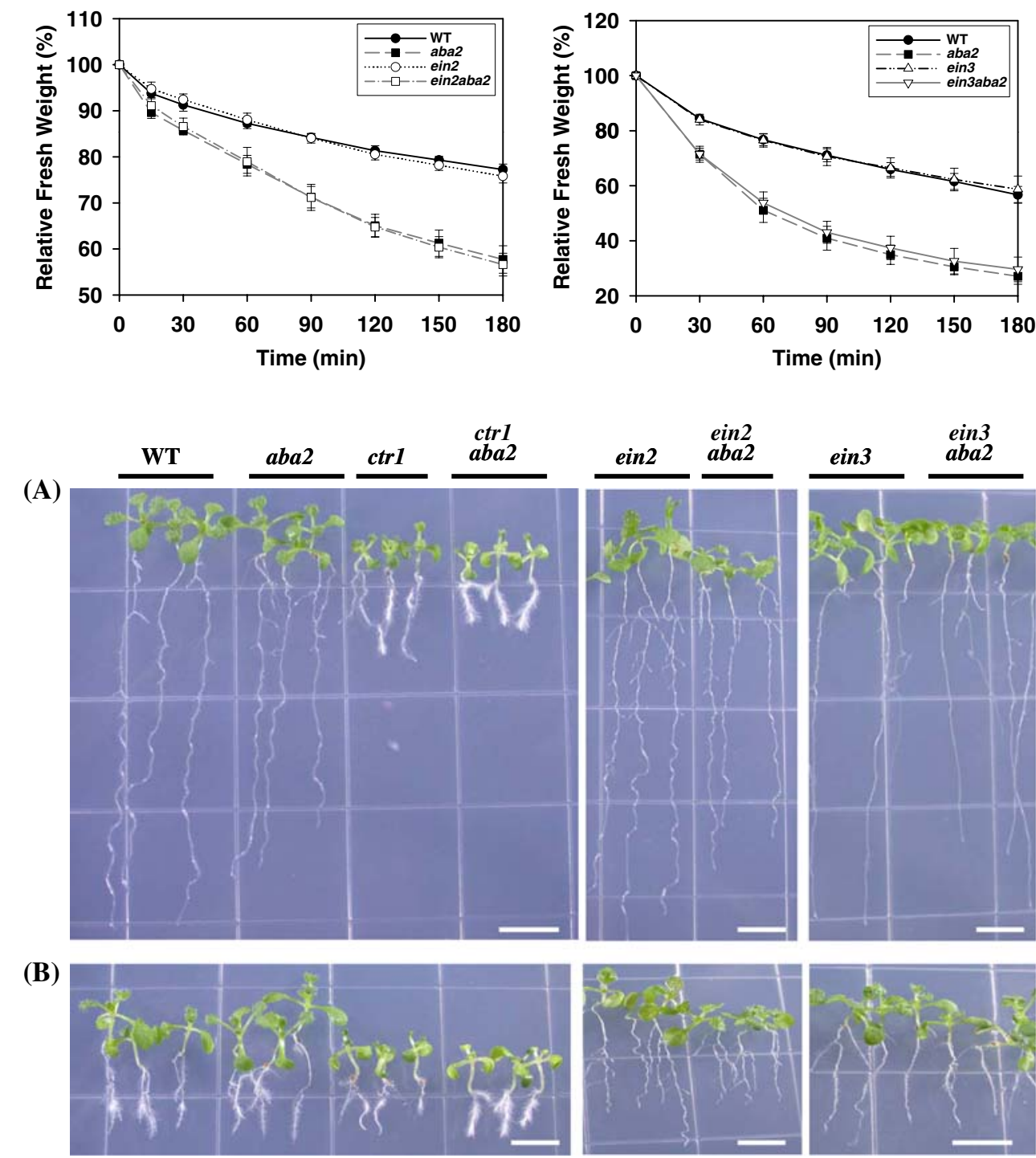
As the ctrl and ein mutants were isolated according to their ethylene triple response under dark conditions, we tested the triple response of the ctrlaba2 and einaba2 double mutants following exposure to ACC in the dark. Our results showed that the wild type and $a b a 2$ were ACC sensitive and had a primary root length that manifested $\sim 87$ and $84 \%$ reductions, respectively, relative to the wild type plants grown on the medium without ACC (Fig. 4B). The primary root in the $a b a 2$ mutant was $28 \%$ shorter than that of the wild-type plant in the absence of ACC (Fig. 4B). The ctrl and ctrlaba2 mutants had a constitutive ethylene triple response when grown on the media with or without ACC. However, ctrlaba2 plants had only $43 \%$ of the primary root length of the ctrl mutant without ACC treatment. Although the ctrl- 1 allele is very strongly associated with the constitutive triple response to ethylene, its primary root showed a $\sim 37 \%$ reduction in length in the presence of ACC, reflecting the fact that ctrl plants are still weakly sensitive to this agent. The ctrlaba2 mutants also had a $27 \%$ reduction in their primary root elongation in the presence of ACC, which was slightly shorter than that in the $c t r l$ single mutant under the same growth conditions. Each of the ein mutants exhibited an ACC insensitive phenotype (Fig. 3A, B) relative to the wild type in the presence of ACC. Similarly, the einaba2 mutants also exhibited this phenotype when grown on the media with or without ACC. Notably, the double mutants all had shorter primary root lengths than their corresponding ein single mutants under the same growth conditions. The reduction in the primary root length in the double mutants, ein2aba2 and ein $3 a b a 2$, was about 31 and $23 \%$ shorter, respectively, than that of the corresponding ein single mutants when grown on the medium without ACC. The primary root lengths in the ein 6 and ein $6 a b a 2$ plants were more sensitive to ACC and showed a $50 \%$ reduction compared to plants grown in the absence of ACC.

In the case of hypocotyls, the wild-type and $a b a 2$ plants showed similar lengths in both the presence and absence of ACC. In the presence of ACC, the wild-type and aba2 hypocotyl lengths were reduced by 52 and $48 \%$, respectively, relative to the lengths measured in the absence of ACC. The ctrl and ctrlaba 2 mutants with a constitutive ethylene triple response phenotype showed similar hypocotyl lengths with a $34 \%$ reduction relative to wild type in the absence of ACC. Upon ACC treatment, both ctrl and ctrlaba2 also showed similar hypocotyl lengths with slight reductions compared to their growth without ACC. The ein and einaba2 mutants revealed ACC insensitive phenotype. The double mutants, ein $2 a b a 2$ and ein $3 a b a 2$, had a slight reduction in their hypocotyl lengths compared with the corresponding single ein mutants when grown on media with or without ACC. However, the ein6aba2 plants had hypocotyl lengths similar to the ein6 single mutant in both
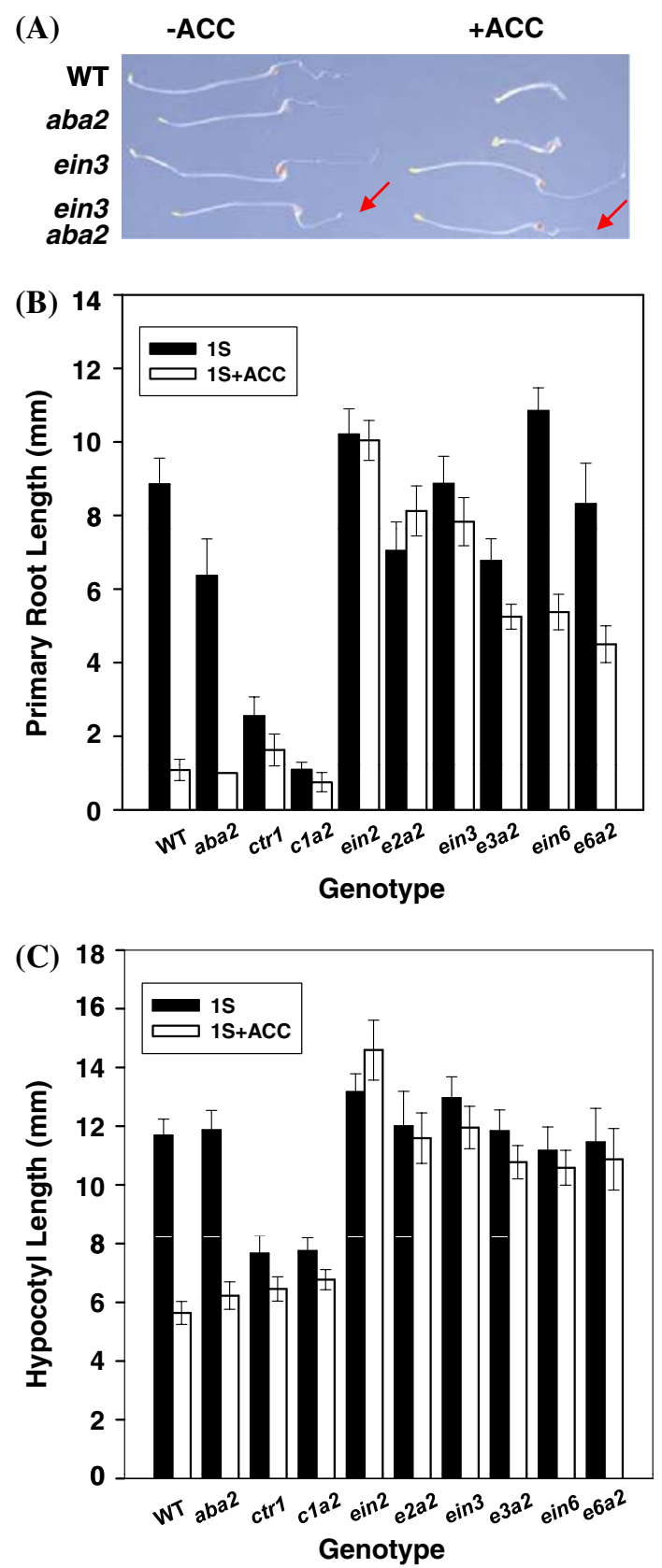

Fig. 4 Comparison of the ethylene response between single and double ABA/ethylene mutants. A-C Seeds were grown on $1 \%$ sucrose agar plates with or without ACC $(10 \mu \mathrm{M})$ treatment for 4 days in the dark. A set of representative phenotypes is shown in (A); the arrows indicate the root tips. The etiolated seedlings were measured for primary root length $(\mathbf{B})$ or hypocotyl length $(\mathbf{C})$, and the data represent the means $\pm \mathrm{SD}, n=8-10$, in one experiment. Three independent experiments were carried out and gave consistent results. c1a2, ctr1aba2; e2a2, ein $2 a b a 2 ;$ e3a2, ein $3 a b a 2 ;$ e6a2, ein6aba 2

the presence and absence of ACC. As ein6 is in the Ler background, whereas $a b a 2$ is in the Col background, we speculated that the ein $6 a b a 2$ double mutant might possess a certain degree of hybrid vigor. We thus further analyzed only the ctrlaba2, ein2aba2, and ein3aba2 plants, 
particularly in the latter two mutants. The data we obtained from the double mutants tested in this study revealed phenotypes that were similar to their respective ethylene single mutants (the constitutive triple response of ctrl and insensitive to ethylene response of ein). Hence, the double mutants conferred both the aba2 phenotype and ctrl or ein phenotype. This indicates that based on their epistatic interaction, the $\mathrm{ABA}$ and ethylene signal transduction pathways act in parallel.

Ethylene sensitivity of root growth between the single and double ABA/ethylene mutants

To address the effects of ethylene upon primary root elongation, our current panel of single and double mutants was tested for their sensitivity to ethylene. As shown in Fig. 5A, the primary roots of the $a b a 2$ mutant were sensitive to ACC and displayed a primary root elongation pattern that paralleled the wild type when the ACC concentrations were below $0.25 \mu \mathrm{M}$. Above this concentration of ACC, the aba2 primary root length showed no difference from the wild type, indicating that $0.25 \mu \mathrm{M}$ ACC is a saturating concentration that is sufficient to inhibit further primary root elongation between $a b a 2$ and wild-type plants. Similarly, the double mutants ein2aba2 and ein $3 a b a 2$ exhibited ACC insensitivity and have short but parallel primary root elongation to their corresponding ein single mutants, i.e., ein 2 and ein 3 , respectively, at all ACC concentrations tested.

Although the $a b a 2$ mutant displayed a shorter primary root than that of the wild type plant, the relative reduction of primary root length in this mutant increased with the ACC concentration up to $0.5 \mu \mathrm{M}$, and this pattern very closely resembled the wild type response (Fig. 5B). These data indicate that the $a b a 2$ mutant and wild-type plants have a near equal sensitivity to ACC, and hence the short primary root observed in the $a b a 2$ mutant does not correlate with ACC sensitivity. In contrast to the above data, the relative reduction in primary root elongation in the ein $2 a b a 2$ double mutant was less altered and resembled the single mutant ein 2 . The double mutant ein $3 a b a 2$ exhibited a very slight increase in its relative reduction of primary root elongation following exposure to ACC and displayed a pattern that paralleled the ein 3 single mutant. These data lend further support to our contention that the double mutants are insensitive to ACC and suggest their shortened primary roots relative to the corresponding single mutants not due to a higher ACC sensitivity. In the case of
Fig. 5 Analysis of ethylene sensitivity between single and double ABA/ethylene mutants. A-C Seeds were grown on $1 \%$ sucrose agar plates

supplemented with a series of different concentrations of ACC for 4 days in the dark. The resulting etiolated seedlings were then subjected to length measurements of their primary roots (A) or hypocotyls (C). In (B), the primary roots of ethylene-treated seedlings were normalized to a control without ACC treatment. D Seeds were grown on $1 \%$ sucrose agar plates supplemented with AVG, $\mathrm{ABA}$, or AVG and ABA for 4 days under dark conditions. Subsequently, the etiolated seedlings were subjected to measurements of their primary root length. The data shown represent the means $\pm \mathrm{SD}$, $n=8-10$. Three independent experiments were carried out and gave consistent results. WT, wild type; ACC, 1-

aminocyclopropane-1carboxylic acid; AVG, L- $\alpha-$ (2-aminoethoxyvinyl)glycine hydrochloride
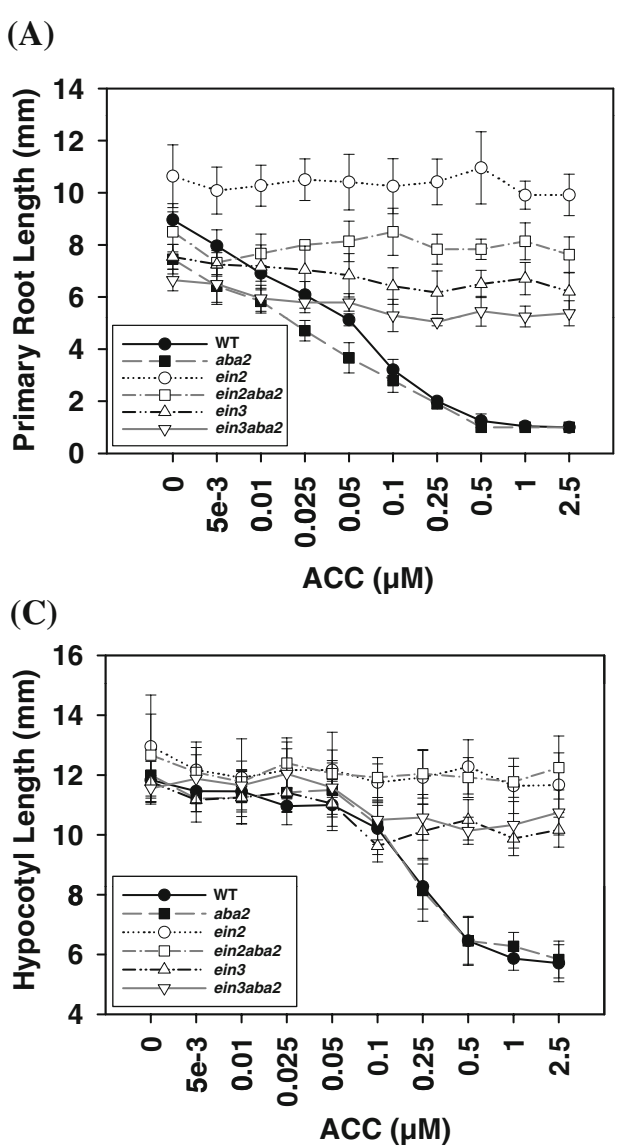

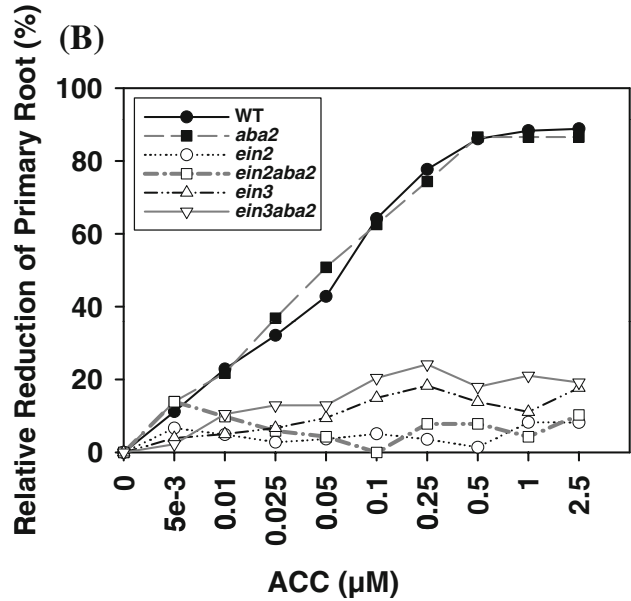

(D)

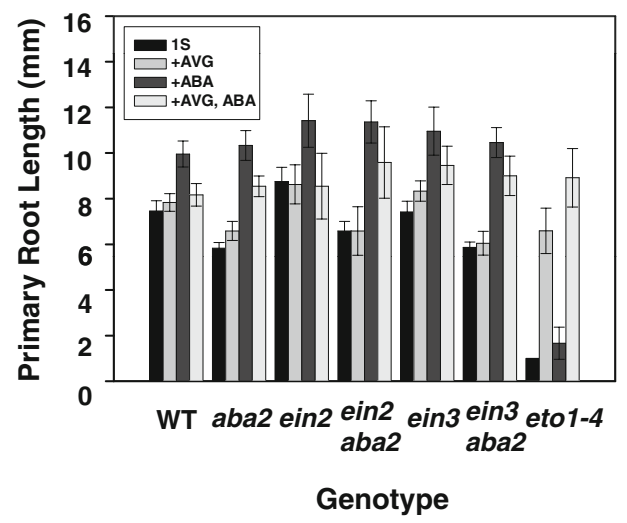


hypocotyl lengths, both the aba2 and wild-type plants had shorter hypocotyls when treated with an ACC concentration over $0.1 \mu \mathrm{M}$, and both displayed a similar ACC sensitivity pattern. However, the ein2 versus ein $2 a b a 2$ and ein3 versus ein $3 a b a 2$ comparisons revealed a very similar and near parallel pattern of relative hypocotyl lengths for all ACC concentrations tested (Fig. 5C), again reflecting the nature of ACC insensitivity in ein and einaba2 mutants.

In our previous study, we demonstrated that the short roots in the aba2 mutant are due to the ABA deficit when grown in light (Lin et. al. 2007). It is thus likely that the short roots observed in the etiolated aba2 and double mutants are due to the lack of ABA. To answer this question, the aba2, ein2aba2, and ein3aba2 mutants were cotreated with ABA and AVG (aminoethxyvinylglycine), an ethylene biosynthesis inhibitor. As shown in Fig. 5D, the aba 2 mutant developed shorter primary roots than the wild type, and similar results were also observed for the ein2aba2 and ein3aba2 double mutants relative to their corresponding ein 2 and ein 3 single mutants. The ethylene overproduction 1-4 (eto1-4) mutant displayed appreciably high levels of ethylene and a pronounced ethylene triple response when grown in dark conditions (Kieber et al. 1993). Upon the exogenous application of the ethylene biosynthesis inhibitor AVG, the wild-type, aba2, ein2, ein2aba2, ein3, and ein3aba2 plants did not showed much change in their primary root lengths. However, the etol-4 mutant appeared to have restored lengths to wild-type levels (Fig. 5D). These data indicated that the short primary root observed in the aba2, ein2aba2, and ein3aba2 mutants does not correlate with the endogenous ethylene contents, whereas the short primary root in the etol-4 mutant is mainly due to the high levels of ethylene. Moreover, the application of ABA to the growth medium promoted the primary root elongation of the $a b a 2$, ein2aba2, and ein3aba2 mutants and caused a slight lengthening of the primary root compared to the plants with no ABA treatment. However, the primary root of the etol-4 mutant, in spite of the slight induction in its elongation following ABA treatment, remained very short relative to the wild type. The restoration of primary root elongation to normal levels was also observed following the addition of both AVG and ABA into the growth medium, although a slight reduction of primary root elongation was still observed compared with the addition of ABA alone. This suggests that the presence of ethylene at low endogenous concentrations is required for $\mathrm{ABA}$ to function in promoting root elongation. However, the eto $1-4$ mutant grown in the presence of AVG and ABA displayed a longer primary root elongation than with the AVG treatment only. Presumably, the etol-4 plants might have a residual amount of ethylene production in the presence of AVG, which causes the enhancement of primary root elongation by ABA. Taken together, these data demonstrated that the shorter roots observed in the $a b a 2$, ein $2 a b a 2$, and ein $3 a b a 2$ mutants are the result of an ABA deficit, whereas the short roots in the eto $1-4$ mutant are mainly due to the overproduction of ethylene.

Effects of ABA and ethylene on seed germination and postgermination seedling growth in Arabidopsis

As shown in Fig. 6A, when grown on $2 \%$ glucose agar plates the $c t r l$ and $a b a 2$ mutants, respectively, possessing a constitutive ethylene triple response and an ABA deficiency, displayed earlier seed germination than wild-type plants within the first 3 days of germination. In the comparison between $c t r 1$ and $a b a 2$, the germination in the $a b a 2$ mutant was earlier, and in the case of the ctrlaba2 double mutant there was a slightly earlier germination than that of the aba 2 mutant. These data suggest that the combination of the aba2 and ctrl alleles reveals no additive effect upon seed germination. The ein2 mutant with a high ABA content (Ghassemian et al. 2000; Wang et al. 2007) displayed a significant germination delay relative to the wild type. Thus, the removal of ABA, as observed in the ein2aba2 double mutant, considerably promoted seed germination in a manner that resembled the ctrl mutant. The ein3 mutant, corresponding to a weak allele in terms of ethylene insensitivity, had a seed germination profile that was similar to the wild type. The block upon ABA biosynthesis in the ein3aba2 double mutant enhanced the seed germination pattern so that it more closely resembled that of $a b a 2$. When grown on $4 \%$ glucose agar plates, the onset of seed germination of all genotypes was greatly delayed, particularly in the ein2, ein3, and wild-type plants (Fig. 6B). In general, the germination pattern on $4 \%$ glucose was found to be similar to that on $2 \%$ glucose with only a slight variation. Seed germination was also examined for two other ethylene-related mutants, etrl-1 and eto 1-4, because of the nature of their ethylene insensitivity and overproduction, respectively. The results showed that both etr $1-1$ and ein 2 had a seed germination delay, but this was enhanced in the etol-4 and ctrl mutants (Fig. 6C). These data lend further support to the promotion of seed germination by an increase of ethylene signaling and/or production. Similar results were also observed when the seeds were grown on $4 \%$ glucose (Fig. 6D).

To examine the effects of ABA and ethylene upon early seedling growth, our panel of single and double ABA/ ethylene mutants was grown on glucose media with/or without ABA and ACC added. The use of the glucose assay in this study made it easy to see the interactions between endogenous ABA and ethylene because ABA biosynthetic genes are induced, and its biosynthesis is increased in the presence of glucose (Cheng et al. 2002). As shown in 
Fig. 6 Antagonistic effects of $\mathrm{ABA}$ and ethylene upon seed germination. A-D Seeds were grown on $2 \%$ (A and $\mathbf{C}$ ) or $4 \%$ (B and D) glucose for the periods indicated. Radicle emergence over $1 \mathrm{~mm}$ is referred to as germination. Germination rates were counted as germinated seeds/total germinated seeds at day 10 . The results shown are the means \pm SD of three independent experiments using different seed batches and giving consistent results, each with 100-150 (A and B) or 100 $(\mathbf{C}$ and $\mathbf{D})$ seeds
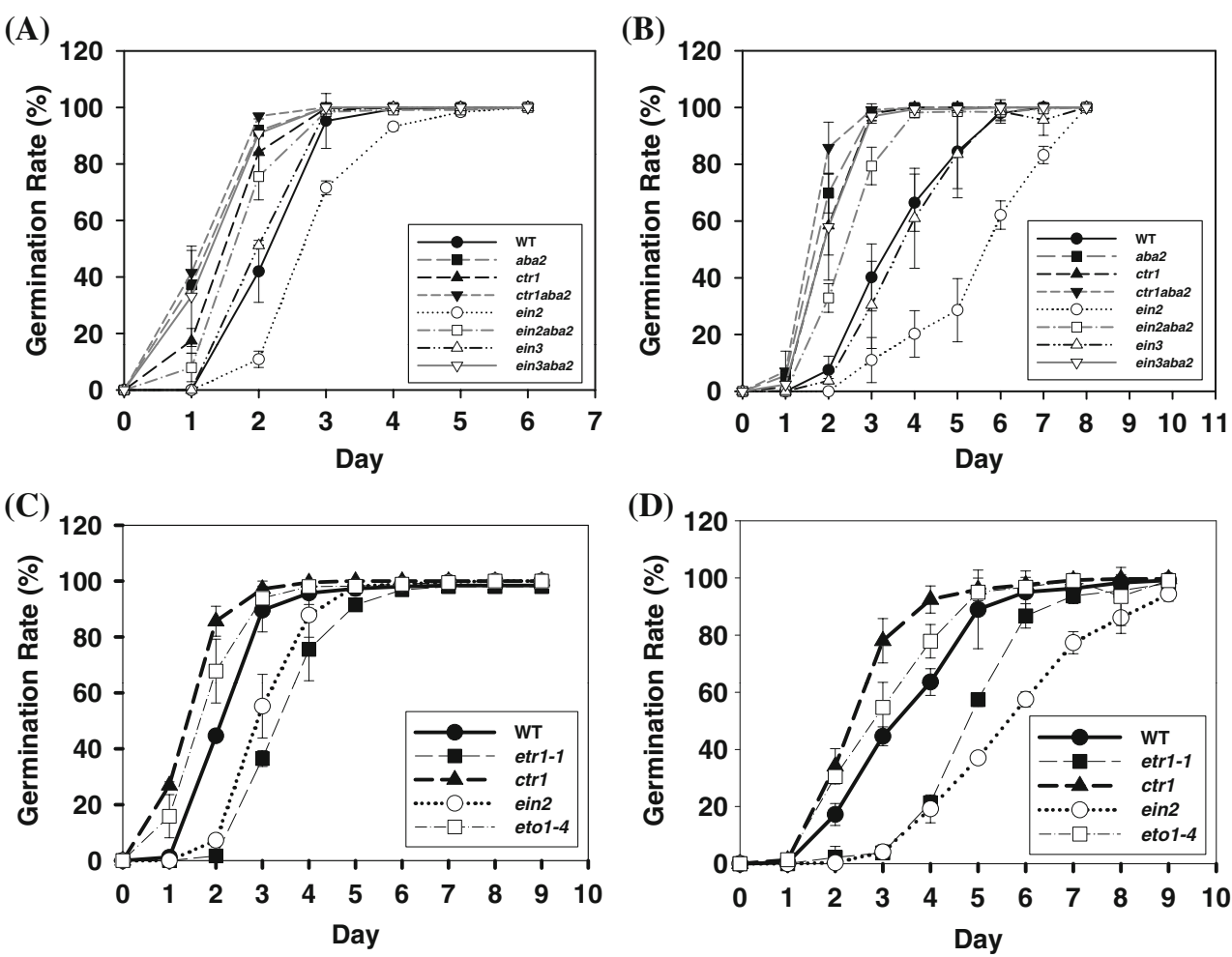

Fig. 7, wild-type plants displayed postgermination developmental arrest when grown on $6 \%$ glucose agar plate for 10 days, whereas the $a b a 2$ mutant grew with expanded cotyledons and greening leaves, a typical glucose insensitive (gin) phenotype. ctrl also showed a gin phenotype despite having a smaller plant size than aba2. A gin phenotype was also observed in the ctrlaba 2 double mutant. In contrast, ein 2 showed developmental arrest on $4 \%$ glucose, which is a glucose oversensitive (glo) phenotype. The ein 3 mutant had a weak glo phenotype and a smaller plant size than the wild-type plants on $4 \%$ glucose concentration. The glo phenotype observed in the ein2 and ein 3 plants reverted to a gin phenotype in the ein2aba 2 and ein $3 a b a 2$ double mutants because of the presence of the $a b a 2$ locus and thus an ABA deficit in these plants. In the presence of ACC $(10 \mu \mathrm{M})$, the developmental arrest observed in wild-type and ein 3 seedlings grown on $6 \%$ glucose disappeared, and a gin phenotype was evident. However, the ein2 mutant
Fig. 7 Effects of ABA and ethylene upon early seedling growth. Seeds were grown on 2 , 4 , or $6 \%$ glucose agar plates supplemented with or without ACC $(10 \mu \mathrm{M})$ or ABA $(100 \mathrm{nM})$ for 10 days in light. Three independent experiments were performed, each with $\geq 30$ seedlings and gave consistent results

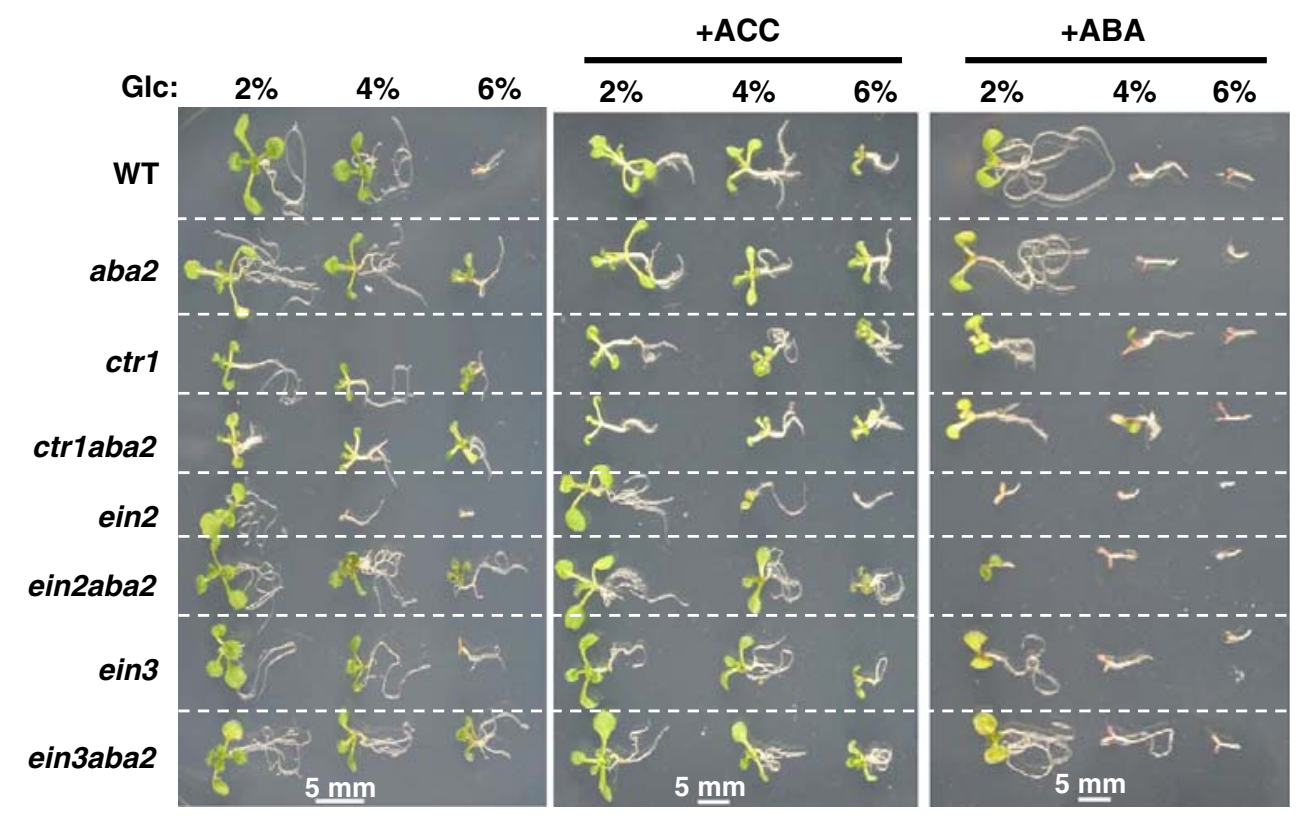


still displayed a developmental arrest on $6 \%$ glucose. In contrast, the developmental arrest in the wild type first appeared at a $4 \%$ glucose concentration in the presence of ABA $(0.1 \mu \mathrm{M})$. Under such growth conditions, the ABAdeficient mutant aba2 reverted to a wild-type phenotype and also showed developmental arrest at $4 \%$ glucose. Similarly, the ein2, ein3, ein2aba2, and ein3aba2 seedlings also exhibited developmental arrest at $4 \%$ glucose in the presence of ABA. It is noteworthy that the ein 2 mutant started to show arrested seedlings at $2 \%$ glucose. ctrl and ctrlaba2 retained a gin phenotype at $4 \%$ glucose in the presence of ABA, but not at $6 \%$ glucose. Collectively, these data suggest that ethylene promotes seed germination and postgermination seedling development, whereas ABA inhibits these processes.
Analysis of the gene expression profile of the $a b a 2$, ein2, and etr $1-1$ mutants

To further study the components that might be involved in the antagonistic interaction between ABA and ethylene, we analyzed the gene expression profile of the aba2, ein2, and etr1-1 mutants using Affymetrix ATH1 GeneChip analysis. These mutant plants were grown on $1 \%$ sucrose agar plates for 12-14 days. Thus, in this study the gene expression profile is mediated by the endogenous ABA and ethylene interaction without exogenous application of hormones or stress. Our results showed that in the aba2 mutant, there were 323 genes (188 up and 135 downregulated genes) with at least a 2-fold change in expression compared with the wild type (Table 1 and Supplementary Table S1; raw

Table 1 ABA- and ethylene-related gene expression in the aba2, etrl, and ein2 mutants

\begin{tabular}{|c|c|c|}
\hline Locus ID & Description $^{\mathrm{a}}$ & Signal fold change ${ }^{b}$ \\
\hline \multicolumn{3}{|c|}{ Significant changes of gene expression in the $a b a 2$ mutant } \\
\hline At1g12010 & Similar to 1-aminocyclopropane-1-carboxylate oxidase & 2.60 \\
\hline At2g20880 & Ethylene-responsive transcription factor ERF053 (ERF053) & 10.2 \\
\hline At1g52340 & ABA deficient 2, ABA2; glucose insensitive 1, GIN1 & 0.48 \\
\hline At5g59220 & Protein phosphatase $2 \mathrm{C}$, putative; response to $\mathrm{ABA}$ stimulus & 0.33 \\
\hline At5g15960 & Cold and ABA inducible protein KIN1 & 0.31 \\
\hline At2g29090 & CYP707A2: cytochrome P450, family 707, subfamily A, polypeptide 2 & 0.21 \\
\hline \multicolumn{3}{|c|}{ Significant changes of gene expression in the etrl mutant } \\
\hline At4g37770 & ACS8, 1-Amino-cyclopropane-1-carboxylate synthase 8 & 7.85 \\
\hline At2g40940 & ERS1, Ethylene response sensor 1 & 0.46 \\
\hline At1g77330 & Similar to 1-Aminocyclopropane-1-carboxylate oxidase & 0.44 \\
\hline At1g04310 & ERS2, Ethylene response sensor 2 & 0.25 \\
\hline At3g16770 & Ethylene-responsive element binding protein, ERF72 & 0.19 \\
\hline At5g25350 & EBF2, EIN3-binding $\mathrm{F}$ box protein 2 & 0.22 \\
\hline At4g26080 & ABI1, Abscisic acid insensitive 1 & 0.41 \\
\hline At2g29090 & CYP707A2: cytochrome P450, family 707, subfamily A, polypeptide 2 & 0.18 \\
\hline \multicolumn{3}{|c|}{ Significant changes of gene expression in the ein 2 mutant } \\
\hline At4g37770 & ACS8, 1-Amino-cyclopropane-1-carboxylate synthase 8 & 3.87 \\
\hline At3g23150 & ETR2, Ethylene response 2 & 0.35 \\
\hline At2g40940 & ERS1, Ethylene response sensor 1 & 0.35 \\
\hline At5g03280 & EIN2, Ethylene insensitive 2 & 0.23 \\
\hline At1g04310 & ERS2, Ethylene response sensor 2 & 0.14 \\
\hline At5g25350 & EBF2, EIN3-binding $\mathrm{F}$ box protein 2 & 0.10 \\
\hline At5g67030* & ABA1, Abscisic acid deficient 1 & 0.95 \\
\hline At3g14440 & NCED3, Nine-cis-epoxycarotenoid dioxygenase 3 & 2.38 \\
\hline At5g15960 & Cold and ABA inducible protein KIN1 & 2.45 \\
\hline At3g02480 & ABA-responsive protein-related & 3.81 \\
\hline
\end{tabular}

a The bold type stands for genes related to ABA function, whereas the other represents the ethylene-related genes

b Signal fold changes in the mutants are the means of two biological experiments and normalized to its corresponding wild type. Genes are referred to have significant change with the following criteria: the expression signal over 100 at either wild type or mutants, each experiment with signal fold change over 1.92 and at least 2-fold changes on average of two biological experiments. The raw data are available in GEO database with an accession no. GSE 12715

* Indicates no significant signal fold change 
data with a GEO accession no. GSE12715). Of these genes, only one identified transcript, ACC oxidase (ACC, Atlg12010), is involved in ethylene biosynthesis (Table 1). Hence, none of the genes involved in the primary ethylene signal transduction pathway were found to be significantly regulated in this analysis. Another ethylene response gene, ETHYLENE RESPONSE FACTOR (ERF053; At2g20880), was also found to be upregulated. The $A B A 2$ transcript levels in the $a b a 2$ mutant were downregulated by 2.1 -fold. Two ABA-inducible genes, protein phosphatase 2C (At5g59220) and KIN1 (At5g15960), were downregulated by $\sim 3$-folds. CYP707A2, encoding an ABA $8^{\prime}$-hydroxylase, was shown to be reduced by $\sim 4.8$-fold (Table 1 ), presumably due to the ABA substrate deficiency in this mutant. In addition to ABA- and ethylene-related genes listed in Table 1, genes that had an altered expression in the $a b a 2$ mutant and participated in hormonal biosynthesis or signaling, and stress and ubiquitination regulation are listed in Supplementary Table S1. For example, the expression of gibberellin 20 oxidase (GA20ox, At5g51810), an important enzyme in the biosynthesis of the bioactive GA compound $\mathrm{GA}_{4}$ in Arabidopsis (for review, see Hedden and Phillips 2000), was increased by $\sim 4.7$-fold. As ABA is a stress hormone, various genes in the $a b a 2$ mutant were found to be abiotic or biotic stress-related. Also, genes likely involved in protein modification (ubiquitination) were also observed in aba2 (Supplementary Table S1).

In the ein 2 mutant, a total of 229 genes had altered expression, with 113 up and 116 downregulated genes (Table 1 and Supplementary Table S1). Of these, several genes involved in ethylene biosynthesis, signaling, and response, such as ACS8, ETR2, ERS1, ERS2, EIN2, and $E B F 2$, were significantly regulated. EIN2 transcripts in this mutant were downregulated by $\sim 4.3$ fold. However, only one ABA biosynthesis gene, NCED3, was appreciably enhanced by 2.4 fold (Table 1). Two other ABA-responsive genes, KINI and At3g02480, were also induced in the ein2 mutant. Several genes with altered expression were involved in hormonal biosynthesis or signaling of gibberellin, cytokinin, jasmonic acid, and salicylic acid. It is noteworthy that three JA-responsive marker genes, vegetative storage proteinl (VSP1, At5g24780), lipoxygenase (LOX2, At3g45140), and thionin (THI2.1, AT1g72660), which are involved in the wounding response, were upregulated in the ein 2 mutant, but not in etrl. Altered genes involved in stress (abiotic and biotic) response and protein ubiquitination were also observed in ein2 (Supplementary Table S1).

To then examine whether the increased expression of NCED3 in the ein 2 mutant is a common feature of ethylene-signaling defective mutants, the etrl-1 mutant was analyzed in the same way. The results showed that in the etrl-1 mutant, 191 genes (94 up and 97 downregulated) were differentially expressed compared to the wild type (Table 1 and supplementary Table S1). Like ein2, the etr 1 mutant had several ethylene biosynthesis, signaling, and response genes that showed significant changes in their expression profiles (Table 1), such as ACS8, ACO-like (At1g77330), ERS1,ERS2, ERF72, and EBF2. etrl-1 is a point mutation showing no conceivable change in its transcript. However, in the case of ABA-related genes, only two genes were significantly changed; $A B I 1$, involved in ABA signaling, was downregulated by $\sim 2.4$ fold, and CYP707A2 was reduced by 5.6 fold. In addition, many other biosynthesis or signal genes of hormones, such as auxin, cytokinin, gibberellin, jasomonate, and brassinosteroid, were also changed in their expression in the etrl mutant. It is noteworthy that at least five auxin responsive and jasmonate biosynthesis or signaling genes were upregulated in etrl. Similarly, several altered genes in this mutant were regulated by abiotic and biotic stress and ubiquitination (Supplementary Table S1). Genes with altered expression listed in Table 1 were further validated by semiquantitative RT-PCR, and the results were generally in agreement with the microarray data, except for a few genes showing variation (Fig. 8A); several genes listed in Table S1 were also verified and are shown in Fig. S2.

Measurement of $\mathrm{ABA}$ and ethylene contents in the ABA/ethylene mutants

As ACO (At1g12010) expression is upregulated in the aba2 mutant, it is conceivable that this mutant might have higher ethylene content than the wild type, as evidenced by LeNoble et al. (2004). To confirm whether the higher ethylene level in $a b a 2$ is because of the upregulation of this $A C O$, we thus measured ethylene in these plants using HP-GC and found that the aba2 mutant had an $\sim 82 \%$ higher ethylene content than the wild type (Fig. 8B) and that the acoaba 2 double mutant had $\sim 65 \%$ higher levels of ethylene. The aco mutant only contained $\sim 85 \%$ of the wildtype ethylene level. These results suggest that the upregulation of $A C O$ in the $a b a 2$ mutants only contributes to the partial induction of ethylene. The other ACS or ACO genes may contribute to the increase in the ethylene levels in the aba2 mutant, but they might be regulated at the translational or post-translational levels, and thus not be detectable by microarray. Interestingly, and despite the higher levels of endogenous ABA described previously (Ghassemian et al. 2000; Wang et al. 2007), the ein2 mutant also was found to accumulate a 4.2-fold higher ethylene content than the wild type. The etr $1-1$ mutant had a $20 \%$ increased ethylene content compared with the wild type. The ctrl mutant only contained $\sim 53 \%$ of the wild-type ethylene level, whereas the etol-4 mutant accumulated ethylene at a $\sim 14.2$-fold higher level than the wild type (Fig. 8B). 
Fig. 8 Validation of ABA- and ethylene-related gene expression, and measurement of the ethylene and ABA contents in the ABA/ethylene mutants. A Validation of the GeneChip data by semiquantitative RT-PCR. Black bars represent means of normalized GeneChip signal of two independent experiments.

Grey bars represent means \pm SD of two independent experiments of semiquantitative RT-PCR. Each experiment was duplicated. The genes tested here were derived from Table 1. B, C Analysis of ethylene contents. Seeds were grown on $1 \%$ sucrose agar medium under dark conditions for 3 days (B) or 4 days (C).

The etiolated seedlings were subsequently measured for ethylene content. The data shown are the means \pm SD of two experiments, each with a triplicate and giving the consistent results. D ABA contents. Seeds were grown on $1 \%$ sucrose agar plates for 14 days. Then, the seedlings were harvested and subjected to the ABA assay. The values are the means $\pm \mathrm{SD}$ of three independent experiments, each with a duplicate and giving consistent results. $* P<0.05$ $(P=0.049)$, Student's $t$ test. ** $P<0.01(P=0.005)$,

Student's $t$ test. ACO, ACC oxidase (At1g12010); UBQ ubiquitin, WT wild type

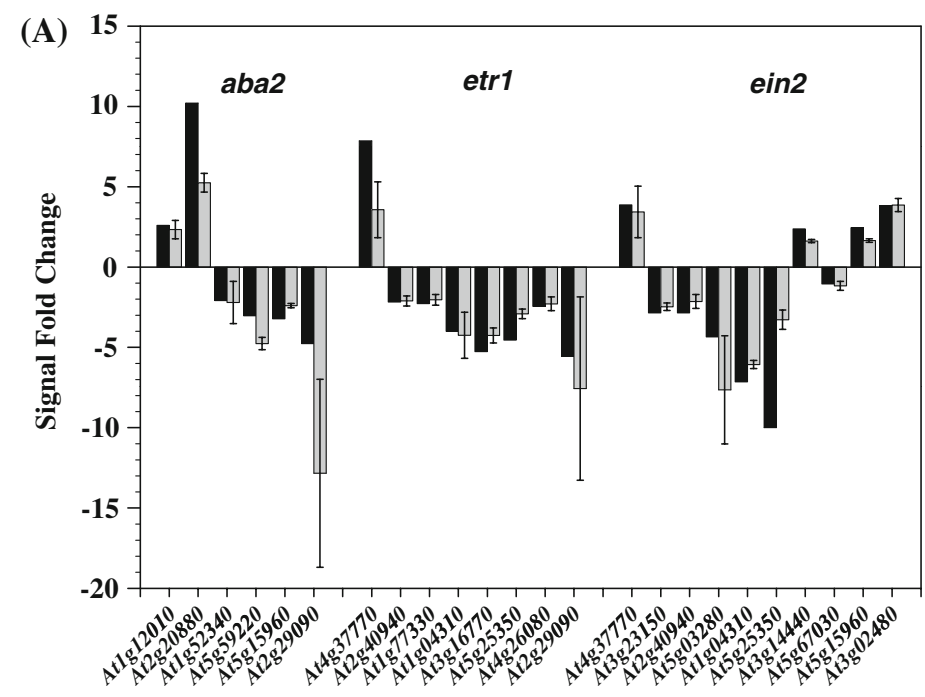

Gene Locus

(B)

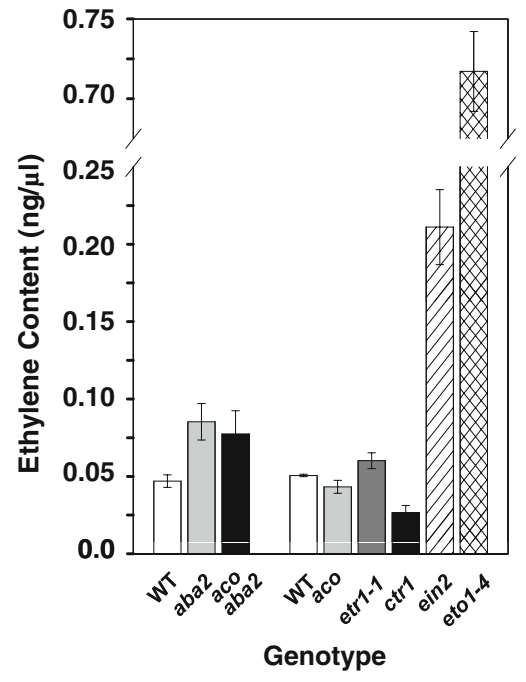

Comparison of ethylene levels among these ABA/ethylene mutants revealed that the double mutants ctrlaba2, ein $2 a b a 2$, and ein $3 a b a 2$ had slightly higher ethylene levels than their corresponding single ethylene mutants $c t r 1$, ein2, or ein3 (Fig. 8C). The higher ethylene contents in these double mutants were attributable to the aba2 mutant allele showing higher ethylene production. For ABA contents, the aba2 mutant ordinarily contains $\sim 20-30 \%$ of wildtype ABA levels (Lin et al. 2007). The ein2 mutant was found to have a $\sim 1.8$-fold higher level than the wild type; the etrl-1 had a $\sim 20 \%$ higher level of ABA than that of the wild type (Fig. 8D). These data indicate that $A B A 2$ is a negative regulator of ethylene biosynthesis. Similarly, EIN2 was revealed as a negative regulator controlling ABA biosynthesis, whereas ETR1-1 was found to be a positive regulator controlling $A B I 1$ and $C Y P 707 A 2$ expression. The antagonism between $\mathrm{ABA}$ and ethylene and their possible interaction in seed germination and early seedling growth is illustrated schematically in Fig. 9.

\section{Discussion}

Parallel signaling between ABA and ethylene and their effects on shoot and root growth

There is now compelling evidence that $\mathrm{ABA}$ and ethylene are very closely involved in various aspects of plant growth, development, and stress response. For instance, these two hormones have contrasting effects on stomatal aperture (Tanaka et al. 2005), hyponastic growth (Benschop et al. 2007), seed germination (Beaudoin et al. 2000; Ghassemian et al. 2000), postgermination seedling growth (Zhou et al. 1998), defense gene expression, and disease 


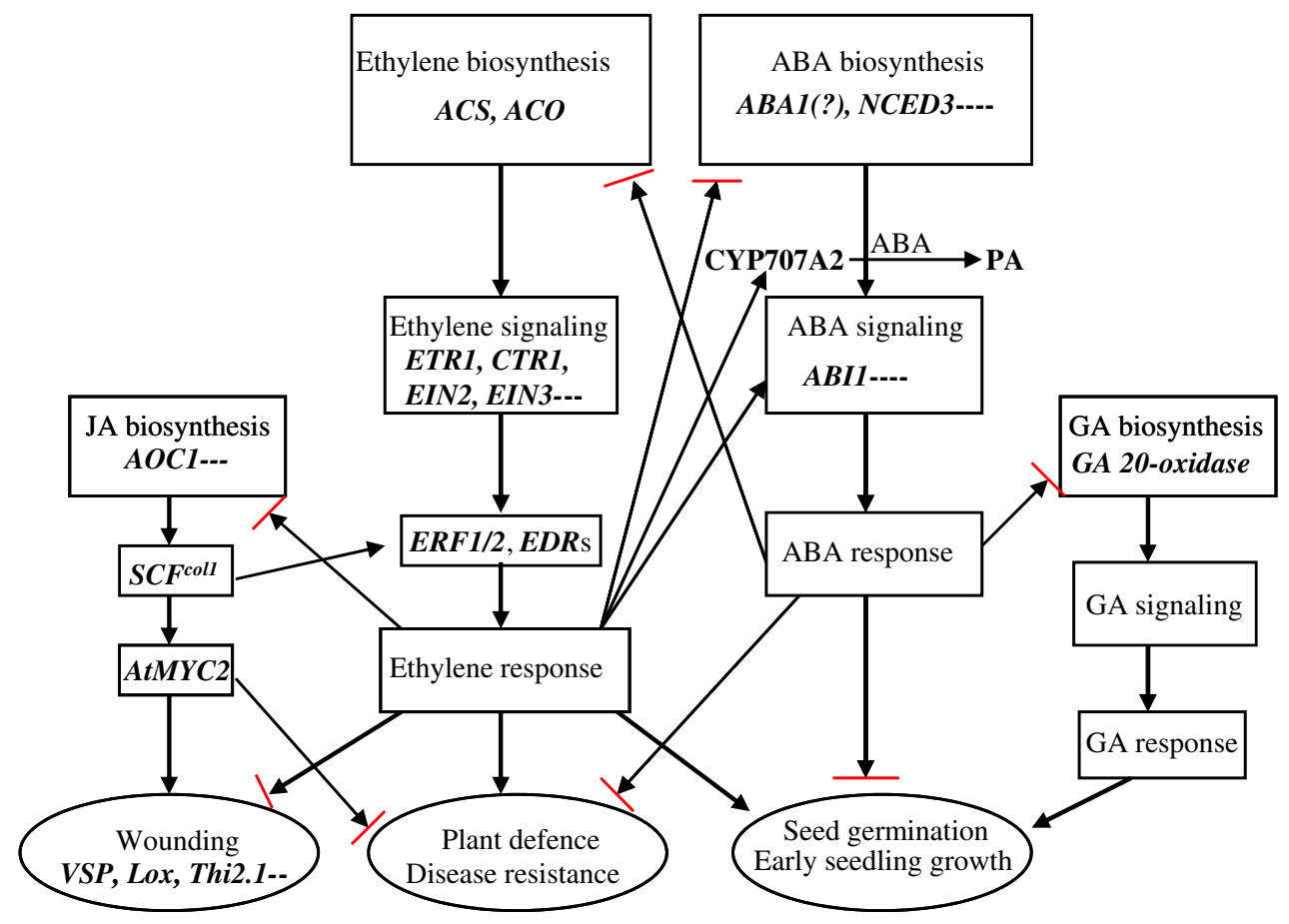

Fig. 9 Schematic representation of a possible mechanism underlying the antagonistic interaction between the ABA and ethylene-signaling pathways. The model depicted is based on genetic epistasis analysis, microarray data, and the measurement of the ethylene and ABA contents of the mutants under study. In general, the ABA and ethylene signal transduction pathways appear to act in parallel with their antagonistic effects upon seed germination and early seedling growth. Moreover, $\mathrm{ABA}$ and ethylene in the $a b a 2$, ein2, and etrl mutant

resistance (Anderson et al. 2004; De Paepe et al. 2004). They also confer synergistic inhibition of root growth (Beaudoin et al. 2000; Ghassemian et al. 2000). Despite the physiological significance of $\mathrm{ABA}$ and ethylene, it was unknown whether there were any convergent points between the corresponding hormone signal transduction pathways or if they functioned only in parallel. To address this issue, we generated double mutants by crossing ethylene mutants (ctr1, ein2, ein 3 , and ein6) with the $a b a 2$ (or ginl-3) mutant. The genetic hierarchy of the components of the ethylene signaling pathway has been well established by epistasis analysis of ethylene mutants (Roman et al. 1995). The essential components, ETR1, CTR1, EIN2, and EIN3, form a largely linear pathway in the early stages of ethylene signal transduction in plants (Bleecker and Kende 2000; Guo and Ecker 2004). Although the precise relationship between EIN6 and other components of the ethylene-signaling pathway has remained obscure, biochemical data suggested that EIN6 acts upstream of EIN3 (Guo and Ecker 2003). ABA has multiple sites of perception and a complex signaling pathway. In addition, the $A B I$ genes identified to date only respond to parts, but not all, of ABA or stress-signaling pathways. Hence, the seedlings may control the hormonal biosynthesis, catabolism, or signaling of each other to enhance their antagonistic roles. It is noteworthy that ABA signal pathway is parallel to the primary (or early) ethylene pathway upstream of EIN3, whereas it crosstalks with the secondary (or late) stages of ethylene-signaling pathway downstream of EIN3. CYP707A2 is an ABA 8'-hydroxylase that catalyzes the breakdown of ABA into phaseic acid (PA). The JA and ethylene interaction was partially adopted from Adie et al. (2007)

crosstalk between $\mathrm{ABA}$ and ethylene might also be diverse. To simplify the study of the ABA-ethylene interaction, we used the aba2 mutant as it has an ABA deficiency and subsequently influences all ABA-signaling pathways. The null mutant $a b a 2$ has a wilty phenotype and small plant size and is ideal for being able to isolate double mutants and classify the interaction relationship between the ABA and ethylene signal transduction pathways. From previous reports and also the data presented in this study, several lines of evidence now indicate that $\mathrm{ABA}$ and ethylene signalings act in parallel. First, previous studies have demonstrated that the etrlaba2 double mutant exhibits phenotypes that closely resemble aba2 (Zhou et al. 1998; LeNoble et al. 2004). In addition, this double mutant also displayed ethylene insensitivity with no induction of root hairs in the presence of ACC, further suggesting that $\mathrm{ABA}$ and ethylene act largely independently of each other in these phenotypes (Zhou et al. 1998). In our current study, we provided further evidence for the independent relationship between $\mathrm{ABA}$ and ethylene by analyzing the downstream ethylene signaling components, ctrl, ein2, and ein3 (Figs. 1, 2, 3, 4). Second, our current double mutants displayed both wilty phenotype and water-loss kinetics 
similar to $a b a 2$ (Fig. 2). Third, in addition to light-grown conditions, the double mutants showed a constitutive ethylene triple response in ctrlaba 2 or ethylene insensitivity in einaba2 mutants in the dark (Fig. 4). These findings indicate that the double mutants contain both $a b a 2$ and ethylene mutant phenotypes, suggesting that $\mathrm{ABA}$ and ethylene function in parallel at least during primary (or early) signal transduction pathways.

In addition, $\mathrm{ABA}$ and ethylene have differential effects on plant growth and development. In general, a comparison of $a b a 2$ with ein and einaba2 revealed that ABA-deficient mutants $a b a 2$ and einaba2 had wiltiness and small plant size. Similar results regarding ABA and ethylene interaction and their effects on shoot growth have also been reported previously. In $a b a 2-1$, for instance, the shoot growth is substantially inhibited, and the plant ethylene content is higher than that of wild type. However, the aba2-1etr1-1 double mutant showing ABA deficit and ethylene insensitivity substantially, but not completely, restores shoot growth to etrl-1 (LeNoble et al. 2004). Similar inhibition of shoot growth through high ethylene production was also observed in the ABA-deficient flacca and notabilis mutants of tomato (Sharp et al. 2000). These data suggest that the impairment of shoot growth in the ABA-deficient mutants of Arabidopsis or tomato is at least partly attributable to the increased ethylene production (LeNoble et al. 2004). In addition to reduced shoot growth, the $a b a 2$ mutant displayed a short root phenotype that was also observed in maize ABA-deficient mutant $v p 5$. Short root in $v p 5$ can be restored by exogenous application of ABA, or substantially improved by ethylene biosynthesis or signaling inhibitors AVG or STS (silver thiosulfate) (Sharp 2002; Sharp and LeNoble 2002; Spollen et al. 2000). In Arabidopsis, although exogenous application of ABA can restore aba2 short roots to normal root growth, the application of AVG in $a b a 2$ only slightly improved its root growth (Fig. 5). One possibility for this discrepancy is that maize and Arabidopsis might have different ethylene sensitivity in roots. Collectively, these data demonstrate that normal or basal levels of ABA are essential to maintain root growth.

Interaction between $\mathrm{ABA}$ and ethylene in seed germination and early postgermination seedling growth

Previous studies demonstrated that transgenic plants overexpressing the Arabidopsis ABA biosynthesis genes, $N C E D 3$, and $A B A 2$, cause ABA accumulation, an increase in dormancy, seed germination delay, and stress tolerance (Iuchi et al. 2001; Lin et al. 2007). Conversely, ABAdeficient mutants ordinarily display a decrease in dormancy, early seed germination, and stress sensitivity. Notably, in our current studies we tested the effect of endogenous $\mathrm{ABA}$ and ethylene on seed germination and early seedling growth, rather than by adding exogenous phytohormones as reported previously (Ghassemian et al. 2000; Beaudoin et al. 2000). In wild-type seeds, exogenous ethylene does not promote seed germination in the absence of ABA, whereas it enhances seed germination in the presence of ABA (Ghassemian et al. 2000). In planta, however, the overproduction of ethylene in etol-4 and the constitutive triple response in $c t r l$ lead to early seed germination (Fig. 6C, D), particularly in the case of $c t r l$; both etol-4 and ctrl showed little change in ABA sensitivity as compared to the wild type (Supplemental Fig. S1). Although both $a b a 2$ and ctrl promoted seed germination, the effects of an ABA deficit and ethylene constitutive signaling are not additive (Fig. 6). In contrast to etol-4 and ctrl, the ethylene-insensitive mutants ein 2 and etrl showed a seed germination delay and an increased ABA sensitivity (Figure S1; Ghassemian et al. 2000; Beaudoin et al. 2000), presumably due to the accumulation of $\mathrm{ABA}$ in these mutants (Fig. 8; Ghassemian et al. 2000; Chiwocha et al. 2005). However, this is not a common feature of ethyleneinsensitive mutants, because ein 3 showed little change in seed germination relative to the wild type (Fig. 6). Taken together, our data generally demonstrated the presence of an antagonistic interaction between $\mathrm{ABA}$ and ethylene in terms of seed germination.

In addition to seed germination, $\mathrm{ABA}$ and ethylene also show antagonistic interactions in postgermination seedling growth (Zhou et al. 1998). Several ABA deficient and insensitive mutants (abal, aba2, aba3, abi4 and abi5) display a glucose insensitive (gin) phenotype in the presence of $6 \%$ glucose, a concentration that causes postgermination developmental arrest in wild-type plants, but not in these mutants (Zhou et al. 1998; Arenas-Huertero et al. 2000). Similarly, the ethylene mutants, ctrl and etol, also have a gin phenotype (Zhou et al. 1998). In contrast, the ethylene-insensitive mutants, etrl, ein2, and ein3, exhibited the glucose over-sensitive (glo) phenotype at $4 \%$ glucose, a concentration in which wild-type plants can grow steadily, but in which these three mutants show postgermination developmental arrest (Zhou et al. 1998; Yanagisawa et al. 2003). In our current study, ein3 only showed a weak glo phenotype with a small plant size relative to the wild type (Fig. 7), presumably due to differences in the growth conditions. In general, the etrl and ein2 mutants showed a strong glo phenotype that correlated with their ABA accumulation and sensitivity, whereas the gin phenotype was found to be associated with $\mathrm{ABA}$ deficiency/or insensitivity and ethylene overproduction/or ethylene constitutive signaling. Thus, ABA inhibits seed germination and postgermination seedling growth, whereas ACC or ethylene promotes these processes. 
Possible mechanism for the antagonistic interaction between the ABA and ethylene signaling cascades

As mentioned above, the ABA and ethylene signal transduction pathways function in parallel and have antagonistic interaction during seed germination and early seedling growth. Previous studies have shown the gene expression profiles mediated by exogenous application of plant hormones or stress (Van Zhong and Burns 2003; Anderson et al. 2004; De Paepe et al. 2004). However, in our current studies we focused on the role of endogenous ABA and ethylene in Arabidopsis physiological effects and hormone interaction without adding exogenous hormones or stress. Thus, the aba2 mutant with its ABA deficiency and higher ethylene contents than the wild type, might further stimulate, at least in part, seed germination and early seedling establishment. However, the high level of ethylene in this mutant was not related to its short primary root, because the short root was the cause of ABA deficiency (Fig. 5). We speculated that the higher ethylene production in $a b a 2$ was due to the upregulation of the $A C O$ (At1g12010) and other ethylene biosynthetic genes at post-translational levels. As several lines of evidence have demonstrated that ethylene biosynthesis and signaling genes are regulated at post-translational levels in Arabidopsis or tomato (Wang et al. 2002; Guo and Ecker 2003; Potuschak et al. 2003; Yanagisawa et al. 2003; Wang et al. 2004; Kevany et al. 2007), these gene products would not be detectable by microarray. ABA-deficient mutants with higher levels of ethylene production have also been observed in tomato (Tal et al. 1979) and Arabidopsis (LeNoble et al. 2004). Collectively, the evidence to date suggests that ABA may regulate ethylene biosynthesis. It has been long believed that the ABA-GA balance plays a critical role in seed dormancy and germination (Koornneef et al. 1982; for review, see Finkelstein et al. 2008). Hence, the change of ABA levels will alter the GA contents as well. Most recent studies revealed that the mutation of genes involved in carotenoid biosynthesis causes ABA deficiency, but induces GA accumulation in rice (Fang et al. 2008). Supporting evidence was also observed in our current studies. For instance, the lack of $\mathrm{ABA}$ in the $a b a 2$ mutant caused the upregulation of a GA biosynthetic gene, GA20-oxidase (Supplementary Table S1; Fig. S2). Hence, the early seed germination and seedling growth observed in aba2 might predominantly be attributable to the change of the ABAGA balance.

ETR1, an ethylene receptor, plays a negative role in regulating the ethylene signal transduction pathway. The gain-of-function mutation of ETR1 in etrl-1 causes insensitivity to ethylene, a seed germination delay, and an ABA oversensitivity. The etrl-2 mutant also has been shown previously to have higher levels of ABA $(\sim 47 \%)$ than the wild type (Chiwocha et al. 2005). The level of ABA induction for the etrl-1 mutant measured in our current study was increased by $\sim 20 \%$ (Fig. $8 \mathrm{C}$ ) relative to the wild type, but this is not sufficient to account for the severe germination delay and ABA oversensitivity, in contrast to the ein 2 mutant, which has $\sim 2$-fold higher ABA levels (Fig. 8C; Ghassemian et al. 2000; Wang et al. 2007). Our microarray data further revealed that the $A B I 1$ and $C Y B 707 A 2$ (Fig. 8A; Table 1) genes were downregulated in the etrl mutant. As $A B I 1$ is a negative regulator of ABA signaling (Sheen 1998; Gosti et al. 1999), its downregulation might cause $\mathrm{ABA}$ oversensitivity in this mutant. Supporting evidence has been shown that the recessive loss-of-function alleles of ABI1, abil-2, and abil-3, enhance response to $\mathrm{ABA}$ both in seed and vegetative tissues of Arabidopsis (Saez et al. 2006). In addition, CYB707A2 is the key enzyme for ABA catabolism. Mutation of CYB707A2 in the $c y b 707 a 2$ mutant causes hyperdormancy and ABA accumulation in seeds (Kushiro et al. 2004). This is consistent with our current result that the reduction in the expression of the $\mathrm{ABA}$ catabolic enzyme CYB707A2 may give rise to ABA accumulation in the etrl mutant. Taken together, the downregulation of $A B I 1$ and $C Y B 707 A 2$ in the etrl mutant resulted in increased both seed dormancy (or seed germination delay) and ABA sensitivity.

EIN2 plays a central role in mediating the ethylene signal transduction pathway and acts as a node that interacts with jasmonic acid, oxygen radicals, and other stress inducers (Alonso et al. 1999). Similar to the etrl mutant, the ein2 mutant displays a germination delay and a high ABA sensitivity, presumably due to the accumulation of ABA (Fig. 8C; Ghassemian et al. 2000; Wang et al. 2007). The high ABA levels in this mutant are most likely due to the upregulation of NCED3 (Fig. 8A; Table 1), a key enzyme in the ABA biosynthetic pathway. Previous studies have revealed that the high ABA levels in ein2 are associated with the upregulation of ABA1 (At5g67030) (Ghassemian et al. 2000; Wang et al. 2007), conflicting with our current results that showed no change of expression in ABAl (Fig. 8A; Table 1). One reason could be the difference in plant developmental stages, stress treatment, or growth conditions. ein2 with high levels of ethylene production (Fig. 8B) was strongly insensitive to ethylene and showed little change in primary root elongation under saturated ACC treatment conditions (Fig. 4B). In contrast, high ethylene concentration in submerged Rumex Palustris and deepwater rice causes strong growth enhancement, with a rapid inhibition of endogenous ABA biosynthesis through the regulation of NCED expression (Kende et al. 1998; Benschop et al. 2005). These data thus reflect that a functional ethylene signal transduction pathway plays a negative role in mediating ABA biosynthesis through the 
regulation of $N C E D$ expression. Our current data revealed that two ethylene-insensitive mutants, etrl and ein2, contained higher levels of ethylene, whereas the constitutive triple response mutant $c t r l$ had a lower ethylene content, only $\sim 53 \%$ of total wild-type levels. These finding are consistent with a negative feedback regulation of ethylene biosynthesis. The higher levels of ethylene in both etrl and ein2 were most likely due to the upregulation of ACS 8 in these mutants. Furthermore, ein 2 had more ethylene production than etrl did, reflecting that the post-translational event might occur in ACS8 or other ethylene biosynthetic genes in these mutants.

In general, the microarray analysis revealed at least two common features in these mutants. First, ABA and ethylene signaling show a complex interaction with other hormones; hence, the change of one hormonal biosynthesis or signaling pathway alters other hormone-related gene expression, which has been reported previously (Chiwocha et al. 2005; for reviews, see Li and Guo 2007; Finkelstein et al. 2008). Second, ABA and ethylene are closely associated with abiotic and biotic stress. Comparison of microarray data also showed that the ABA-inducible gene KIN1 was ABA-dependent, with its reduction in $a b a 2$ but induction in ein2 (Table 1; Fig. 8A). A similar result was also observed in a cold-regulated gene COR15A (At2g42540) (Table S1; Fig. S2). Two ethylene receptors, ERS1 and ERS2, were both downregulated in etr1 and ein2, whereas ETR 2 expression was only reduced in ein 2 , further consistent with previous report that these three ethylene receptors are ethylene-inducible (Hua et al. 1998). EBF2, encoding an EIN3-binding $\mathrm{F}$ box protein, also had an altered expression pattern in both etr1 and ein2 (Table 1; Fig. 8A). Taken together with the ACS8 upregulation in these two mutants, ethylene may regulate genes involved its own biosynthesis and signal transduction pathway as also described previously (Van Zhong and Burns 2003). Ethylene and JA have well-characterized synergistic effects on plant defense and disease resistance, and an antagonistic interaction in wounding in Arabidopsis. Our current data provided evidence that both etr1 and ein 2 promoted the expression of allene oxide cyclase 1 (AOCl; At3g25760), which catalyzes an essential step in JA biosynthesis. Another gene OPDA reductase 3 (OPR3, At2g06050) required for JA biosynthesis was also stimulated in etrl. These data support that ethylene signaling may negatively regulate JA biosynthesis, and its regulation is differential between etrl and ein2. As both etrl and ein 2 are strong ethylene-insensitive mutants, expression profiles of some genes are expected to be similar to some extent, as partially evidenced by the results described above. However, a substantial level of dissimilarity was observed, as shown in Table 1 and Table S1. For example, the three JAmediated markers, VSP1, LOX2, and THI2.1 (for review, see Adie et al. 2007), in response to wounding were upregulated in ein 2 but not in etrl (supplemental Table S1; Fig. S2), reflecting that these two mutants, at least in part, might have a different pathways in response to wounding.

The possible mechanisms underlying the antagonistic interaction between $\mathrm{ABA}$ and ethylene signaling are illustrated in Fig. 9. By epistasis analysis, we demonstrated parallel signaling between $\mathrm{ABA}$ and ethylene in the primary (or early) signal pathway. As the key components, ETR1, CTR1, EIN2, and EIN3, form a largely linear pathway in the early (or primary) stages of ethylene signal transduction pathway in plants. The activated EIN3 or EIN3-like (EIL) transcription factors may switch expression of ERF or EDF transcription factors, which in turn trigger transcription of secondary (or late) ethyleneresponse genes. These secondary ethylene-response genes are involved in specific branches of the ethylene response pathway downstream of EIN3 (Stepanova and Alonso 2005). Global expression analysis further confirms a wide range of ethylene-response genes involved in various metabolic, signal, developmental, and structural functions (Alonso et al. 2003). Thus, the antagonistic crosstalk in this model will occur via secondary (or late) signal transduction pathways. In addition, seed dormancy and germination are regulated by a combination of environmental and endogenous signals (for review, see Finkelstein et al. 2008). The latter includes the integration of plant hormones, such as $\mathrm{ABA}, \mathrm{GA}$, and ethylene, and the effect of other hormones or factors on seed dormancy and germination cannot be excluded by our current findings.

Acknowledgments We thank Drs. Jen Sheen (Massachusetts General Hospital (MGH), Boston, MA) and Kevin L.-C. Wang (IPMB, Academia Sinica) for helpful suggestions, Miss Jessica Penney for genetic screens and Yanxia Liu for microarray analysis (MGH, Boston, MA). We are also grateful to AGESL (Affymetrix Gene Expression Service Lab, Academia Sinica, Taipei, Taiwan) for undertaking the ATH1 GeneChip analysis, Dr. Kevin L.-C. Wang for etol-4 seeds, and ABRC (Columbus, $\mathrm{OH}$ ) for providing additional ethylene mutant seeds. This work was supported by the Academia Sinica (grant no. AS 91IB1PP to W.-H. C) and the National Science Foundation (US) (IBN-9723610 to J. Sheen).

This work is dedicated to Miss Pei-Chi Lin (1978-2007).

Open Access This article is distributed under the terms of the Creative Commons Attribution Noncommercial License which permits any noncommercial use, distribution, and reproduction in any medium, provided the original author(s) and source are credited.

\section{References}

Adie B, Chico JM, Rubio-Somoza I, Solano R (2007) Modulation of plant defenses by ethylene. J Plant Growth Regul 26:160-177. doi:10.1007/s00344-007-0012-6

Alonso JM, Hirayama T, Roman G, Nourizadeh S, Ecker JR (1999) EIN2, a bifunctional transducer of ethylene and stress responses 
in Arabidopsis. Science 284:2148-2152. doi:10.1126/science. 284.5423.2148

Alonso JM, Stepanova AN, Leisse TJ, Kim CJ, Chen H, Shinn P, Stevenson DK, Zimmerman J, Barajas P, Cheuk R, Gadrinab C, Heller C, Jeske A, Koesema E, Meyers CC, Parker H, Prednis L, Ansari Y, Choy N, Deen H, Geralt M, Hazari N, Hom E, Karnes M, Mulholland C, Ndubaku R, Schmidt I, Guzman P, AguilarHenonin L, Schmid M, Weigel D, Carter DE, Marchand T, Risseeuw E, Borgden D, Zeko A, Crosby WL, Berry CC, Ecker JR (2003) Genome-wide insertional mutagenesis of Arabidopsis thaliana. Science 301:653-657. doi:10.1126/science.1086391

Anderson JP, Badruzsaufari E, Schenk PM, Manners JM, Desmond OJ, Ehlert C, Mackean DJ, Ebert PR, Kazan K (2004) Antagonistic interaction between abscisic acid and jasmonateethylene signaling pathways modulates defense gene expression and disease resistance in Arabidopsis. Plant Cell 16:3460-3479. doi: $10.1105 /$ tpc. 104.025833

Arenas-Huertero F, Arroyo A, Zhou L, Sheen J, Leon P (2000) Analysis of Arabidopsis glucose insensitive mutants, gin5 and gin6, reveals a central role of the plant hormone $\mathrm{ABA}$ in the regulation of plant vegetative development by sugar. Genes Dev 14:2085-2096. doi:10.1101/gad.14.16.2085

Beaudoin N, Serizet C, Gosti F, Giraudat J (2000) Interactions between abscisic acid and ethylene signaling cascades. Plant Cell 12:1103-1115. doi:10.1105/tpc.12.7.1103

Benschop JJ, Jackson MB, Guhl K, Vreeburg RAM, Croker SJ, Peeters AJM, Voesenek LACJ (2005) Contrasting interactions between ethylene and abscisic acid in Rumex species differing in submergence tolerance. Plant J 44:756-768. doi:10.1111/j.1365313X.2005.02563.X

Benschop JJ, Millenaar FF, Smeets ME, Zanten M, Voesenek LACJ, Peeters AJM (2007) Abscisic acid antagonizes ethylene-induced hyponastic growth in Arabidopsis. Plant Physiol 143:1013-1023. doi:10.1104/pp. 106.092700

Bittner F, Oreb M, Mendel RR (2001) ABA3 is a molybdenum cofactor sulfurase required for activation of aldehyde oxidase and xanthine dehydrogenase in Arabidopsis thaliana. J Biol Chem 276:40381-40384. doi:10.1074/jbc.C100472200

Bleecker AB, Kende H (2000) Ethylene: a gaseous signal molecule in plants. Annu Rev Cell Dev Biol 16:1-18. doi:10.1146/ annurev.cellbio.16.1.1

Brady SM, Sarkar SF, Bonetta D, McCout P (2003) The ABSCISIC ACID INSENSITIVE3 (ABI3) gene is modulated by farnesylation and is involved in auxin signaling and lateral root development in Arabidopsis. Plant J 34:67-75. doi:10.1046/j.1365-313X. 2003.01707.x

Brocard-Gifford I, Lynch TJ, Garcia EG, Malhotra B, Finkelstein RR (2004) The Arabidopsis thaliana ABSCISIC ACID INSENSITIVE8 locus encodes a novel protein mediating abscisic acid and sugar responses essential for growth. Plant Cell 16:406-421. doi: $10.1105 /$ tpc. 018077

Cheng W-H, Endo A, Zhou L, Penney J, Chen H-C, Arroyo A, Leon P, Nambara E, Asami T, Seo M, Koshiba T, Sheen J (2002) A unique short-chain dehydrogenase/reductase in Arabidopsis glucose signaling and abscisic acid biosynthesis and functions. Plant Cell 14:2723-2743. doi:10.1105/tpc.006494

Chiwocha SDS, Cutler AJ, Abrams SR, Ambrose SJ, Yang J, Ross ARS, Kermode AR (2005) The etr1-2 mutation in Arabidopsis thaliana affects the abscisic acid, auxin, cytokinin and gibberellin metabolic pathways during maintenance of seed dormancy, moist-chilling and germination. Plant J 42:35-48. doi:10.1111/ j.1365-313X.2005.02359.x

Cutler S, Ghassemian M, Bonetta D, Cooney S, McCourt P (1996) A protein farnesyl transferase involved in abscisic acid signal transduction in Arabidopsis. Science 273:1239-1241. doi:10. 1126/science.273.5279.1239
Dall'Osto L, Cazzaniga S, North H, Marion-Poll A, Bassi R (2007) The Arabidopsis aba4-1 mutant reveals a specific function for neoxanthin in protection against photooxidative stress. Plant Cell 19:1048-1064. doi:10.1105/tpc.106.049114

De Paepe A, Vuylsteke M, Van Hummelen P, Zabeau M, Straeten Van Der (2004) Transcriptional profiling by cDNA-AFLP and microarray analysis reveals novel insights into the early response to ethylene in Arabidopsis. Plant J 39:537-559. doi:10.1111/ j.1365-313X.2004.02156.X

Fang J, Chai C, Qian Q, Li C, Tang J, Sun L, Huang Z, Guo X, Sun C, Liu M, Zhang Y, Lu Q, Wang Y, Lu C, Han B, Chen F, Cheng Z, Chu C (2008) Mutations of genes in synthesis of the carotenoid precursors of ABA lead to pre-harvest sprouting and photooxidation in rice. Plant J 54:177-189. doi:10.1111/j.1365313X.2008.03411.x

Finkelstein RR, Lynch TJ (2000) The Arabidopsis abscisic acid response gene $A B I 5$ encodes a basic leucine zipper transcription factor. Plant Cell 12:599-609. doi:10.1105/tpc.12.4.599

Finkelstein RR, Wang ML, Lynch TJ, Rao S, Goodman HM (1998) The Arabidopsis abscisic acid response locus ABI4 encodes an APETALA2 domain protein. Plant Cell 10:1043-1054. doi:10.1105/tpc.10.6.1043

Finkelstein RR, Gampala SS, Rock CD (2002) Abscisic acid signaling in seeds and seedlings. Plant Cell 14:S15-S45. doi:10.1105/ tpc. 010441

Finkelstein RR, Reeves W, Ariizumi T, Steber C (2008) Molecular aspects of seed dormancy. Annu Rev Plant Biol 59:387-415. doi:10.1146/annurev.arplant.59.032607.092740

Fujii H, Verslues PE, Zhu JK (2007) Identification of two protein kinases required for abscisic acid regulation of seed germination, root growth, and gene expression in Arabidopsis. Plant Cell 19:484-494. doi:10.1105/tpc.106.048538

Ghassemian M, Nambara E, Cutler S, Kawaide H, Kamiya Y, McCourt P (2000) Regulation of abscisic acid signaling by the ethylene response pathway in Arabidopsis. Plant Cell 12:11171126. doi:10.1105/tpc.12.7.1117

González-Guzmán M, Apostolova N, Bellés JM, Barrero JM, Piqueras P, Ponce MR, Micol JL, Serrano R, Rodríguez PL (2002) The short-chain alcohol dehydrogenase ABA2 catalyzes the conversion of xanthoxin to abscisic aldehyde. Plant Cell 14:1833-1846. doi:10.1105/tpc.002477

Gosti F, Beaudoin N, Serizet C, Webb AAR, Vartanian N, Giraudat J (1999) ABI1 protein phosphatase $2 \mathrm{C}$ is a negative regulator of abscisic acid signaling. Plant Cell 11:1897-1909. doi:10.1105/ tpc.11.10.1897

Guo H, Ecker JR (2003) Plant responses to ethylene gas are mediated by $\mathrm{SCF}^{\mathrm{EBF} 1 / \mathrm{EBF} 2}$-dependent proteolysis of EIN3 transcription factor. Cell 115:667-677. doi:10.1016/S0092-8674(03)00969-3

Guo H, Ecker JR (2004) The ethylene signaling pathway: new insights. Curr Opin Plant Biol 7:40-49. doi:10.1016/j.pbi.2003.11.011

Hedden P, Phillips AL (2000) Gibberellin metabolism: new insights revealed by the genes. Trends Plant Sci 5:523-530. doi:10.1016/ S1360-1385(00)01790-8

Hua J, Sakai H, Nourizadeh S, Chen QG, Bleecker AB, Ecker JR, Meyerowitz EM (1998) EIN4 and ERS2 are members of the putative ethylene receptor gene family in Arabidopsis. Plant Cell 10:1321-1332. doi:10.1105/tpc.10.8.1321

Iuchi S, Kobayashi M, Taji T, Naramoto M, Seki M, Kato T, Tabata S, Kakubari Y, Yamaguchi-Shinozaki K, Shinozaki K (2001) Regulation of drought tolerance by gene manipulation of 9-cisepoxycarotenoid dioxygenase, a key enzyme in abscisic acid biosynthesis in Arabidopsis. Plant J 27:325-333. doi:10.1046/ j.1365-313x.2001.01096.x

Johnson PR, Ecker JR (1998) The ethylene gas signal transduction pathway: a molecular perspective. Annu Rev Genet 32:227-254. doi:10.1146/annurev.genet.32.1.227 
Karssen CM, Brinkhorst-van der Swan DLC, Breekland AE, Koornneef $M$ (1983) Induction of dormancy during seed development by endogenous abscisic acid: studies of abscisic acid deficient genotypes of Arabidopsis thaliana (L.) Heynh. Planta 157:158165. doi:10.1007/BF00393650

Kende H, van der Knaap E, Cho H-T (1998) Deepwater rice: a model plant to study stem elongation. Plant Physiol 118:1105-1110. doi:10.1104/pp.118.4.1105

Kevany BM, Tieman DM, Taylor MG, Cin VD, Klee HJ (2007) Ethylene receptor degradation controls the timing of ripening in tomato fruit. Plant J 51:458-467. doi:10.1111/j.1365-313X .2007.03170.x

Kieber JJ, Rothenberg M, Roman G, Feldmann KA, Ecker JR (1993) CTR1, a negative regulator of the ethylene response pathway in Arabidopsis encodes a member of the raf family of protein kinase. Cell 72:427-441. doi:10.1016/0092-8674(93) 90119-B

Koornneef M, Jorna ML, Brinkhoust-van der Swan DLC, Karssen CM (1982) The isolation of abscisic acid (ABA) deficient mutants by selection of induced revertants in non-germination gibberellin sensitive lines of Arabidopsis thaliana L. Heynh. Theor Appl Genet 61:385-393. doi:10.1007/BF00272861

Koornneef M, Hanhart CJ, Hilhorst HWM, Karssen CM (1989) In vivo inhibition of seed development and reserve protein accumulation in recombinants of abscisic acid biosynthesis and responsiveness in Arabidopsis thaliana. Plant Physiol 90:463469. doi:10.1104/pp.90.2.463

Kushiro T, Okamoto M, Nakabayashi K, Yamagishi K, Kitamura S, Asami T, Hirai N, Koshiba T, Kamiya Y, Nambara E (2004) The Arabidopsis cytochrome P450 CYP707A encodes ABA 8'hydroxylases: key enzymes in ABA catabolism. EMBO J 23: 1647-1656. doi:10.1038/sj.emboj.7600121

LeNoble ME, Spollen WG, Sharp RE (2004) Maintenance of shoot growth by endogenous ABA: genetic assessment of the involvement of ethylene suppression. J Exp Bot 55:237-245. doi:10.1093/jxb/erh031

Leung J, Giraudat J (1998) Abscisic acid signal transduction. Annu Rev Plant Physiol Plant Mol Biol 49:199-222. doi:10.1146/ annurev.arplant.49.1.199

Leung J, Merlot S, Giraudat J (1997) The Arabidopsis ABSCISIC ACID-INSENSITIVE 2 (ABI2) and ABII encode homologous protein phosphatase $2 \mathrm{C}$ involved in abscisic acid signal transduction. Plant Cell 9:759-771. doi:10.1105/tpc.9.5.759

Li H, Guo H (2007) Molecular basis of the ethylene signaling and response pathway in Arabidopsis. J Plant Growth Regul 26:106117. doi:10.1007/s00344-007-0015-3

Lin P-C, Hwang S-G, Endo A, Okamoto M, Koshiba T, Cheng W-H (2007) Ectopic expression of ABSCISIC ACID 2/GLUCOSE INSENSITIVE 1 in Arabidopsis promotes seed dormancy and stress tolerance. Plant Physiol 143:745-758. doi:10.1104/ pp. 106.084103

Marin E, Nussaume L, Quesada A, Gonneau M, Sotta B, Hugueney P, Frey A, Marion-Poll A (1996) Molecular identification of zeaxanthin epoxidase of Nicotiana plumbaginifolia, a gene involved in abscisic acid biosynthesis and corresponding to the ABA locus of Arabidopsis thaliana. EMBO J 15:2331-2342

North HM, De Almeida A, Boutin JP, Frey A, To A, Botran L, Sotta B, Marion-Poll A (2007) The Arabidopsis ABA-deficient mutant $a b a 4$ demonstrate that the major route for stress-induced ABA accumulation is via neoxanthin isomers. Plant J 50:810-824. doi:10.1111/j.1365-313X.2007.03094.x

Potuschak T, Lechner E, Parmentier Y, Yanagisawa S, Grava S, Koncz C, Genschik P (2003) EIN3-dependent regulation of plant ethylene hormone signaling by two Arabidopsis $\mathrm{F}$ box proteins: EBF1 and EBF2. Cell 115:679-689. doi:10.1016/S0092-8674 (03)00968-1
Qin X, Zeevaart JAD (2002) Overexpression of a 9-cis-epoxycarotinoid dioxygenase gene in Nicotiana plumbaginifolia increases abscisic acid and phaseic acid levels and enhances drought tolerance. Plant Physiol 128:544-551. doi:10.1104/pp.010663

Roman G, Lubarsky B, Kieber JJ, Rothenberg M, Ecker JR (1995) Genetic analysis of ethylene signal transduction in Arabidopsis thaliana: five novel mutant loci integrated into a stress response pathway. Genetics 139:1393-1409

Rook F, Corke F, Card R, Munz G, Smith C, Bevan MW (2001) Impaired Suc-induction mutants reveal the modulation of sugarinduced starch biosynthetic gene expression by abscisic acid signaling. Plant J 26:421-433. doi:10.1046/j.1365-313X.2001. 2641043.x

Saez A, Robert N, Maktabi MH, Schroeder JI, Serrano R, Rodriguez PL (2006) Enhancement of abscisic acid sensitivity and reduction of water consumption in Arabidopsis by combined inactivation of the protein phosphatases type $2 \mathrm{C}$ ABI1 and HAB1. Plant Physiol 141:1389-1399. doi:10.1104/pp.106.081018

Schwartz SH, Tan BC, Gage DA, Zeevaart JAD, McCarty DR (1997) Specific oxidative cleavage of carotenoid by VP14 of maize. Science 276:1872-1874. doi:10.1126/science.276.5320.1872

Schwartz SH, Qin X, Zeevaart JAD (2003) Elucidation of the indirect pathway of abscisic acid biosynthesis by mutants, genes, and enzymes. Plant Physiol 131:1591-1601. doi:10.1104/pp.102. 017921

Seo M, Koshiba T (2002) Complex regulation of ABA Biosynthesis in plants. Trends Plant Sci 7:41-48. doi:10.1016/S13601385(01)02187-2

Seo M, Peeters AJM, Koiwai H, Oritani T, Marion-Poll A, Zeevaart JAD, Koornneef M, Kamiya Y, Koshiba T (2000) The Arabidopsis aldehyde oxidase 3 (AAO3) gene product catalyzes the final step in abscisic acid biosynthesis in leaves. Proc Natl Acad Sci USA 97:12908-12913. doi:10.1073/pnas.220426197

Sharp RE (2002) Interaction with ethylene: changing views on the role of abscisic acid in root and shoot growth response to water stress. Plant Cell Environ 25:211-222. doi:10.1046/j.13653040.2002.00798.x

Sharp RE, LeNoble ME (2002) ABA, ethylene and the control of shoot and root growth under water stress. J Exp Bot 53:33-37. doi:10.1093/jexbot/53.366.33

Sharp RE, LeNoble ME, Else MA, Thorne ET, Gherardi F (2000) Endogenous ABA maintains shoot growth in tomato independently of effects on plant water balance: evidence for an interaction with ethylene. J Exp Bot 51:1575-1584. doi:10.1093/ jexbot $/ 51.350 .1575$

Sheen J (1998) Mutational analysis of protein phosphatase 2C involved in abscisic acid signal transduction in higher plants. Proc Natl Acad Sci USA 95:975-980. doi:10.1073/pnas.95.3.975

Smalle J, Kurepa J, Yang PZ, Emborg TJ, Baiychuk E, Kushnir S, Vierstra RD (2003) The pleiotropic role of the 26S proteasome subunit RPN10 in Arabidopsis growth and development supports a substrate-specific function in abscisic acid signaling. Plant Cell 15:965-980. doi:10.1105/tpc.009217

Spollen WG, LeNoble ME, Samuels TD, Bernstein N, Sharp RE (2000) Abscisic acid accumulation maintains maize primary root elongation at low water potentials by restricting ethylene production. Plant Physiol 122:967-976. doi:10.1104/pp.122.3.967

Stepanova AN, Alonso JM (2005) Arabidopsis ethylene signaling pathway. Sci STKE 276:1-4. doi:10.1126/stke.2762005cm4

Tal M, Imber D, Erez A, Epstein E (1979) Abnormal stomatal behavior and hormonal imbalance in flacca, a wilty mutant of tomato. V. Effect of abscisic acid on indoleacetic acid metabolism and ethylene evolution. Plant Physiol 63:1044-1048. doi:10.1104/pp.63.6.1044

Tanaka Y, Sano T, Tamaoki M, Nakajima N, Kondo N, Hasezawa S (2005) Ethylene inhibits abscisic acid-induced stomatal closure 
in Arabidopsis. Plant Physiol 138:2337-2343. doi:10.1104/ pp. 105.063503

Van Zhong G, Burns JK (2003) Profiling ethylene-regulated gene expression in Arabidopsis thaliana by microarray analysis. Plant Mol Biol 53:117-131. doi:10.1023/B:PLAN.0000009270.81977.ef

Wang KLC, Li H, Ecker JR (2002) Ethylene biosynthesis and signaling networks. Plant Cell 14:S131-S151. doi:10.1105/ tpc. 001768

Wang KLC, Yoshida H, Lurin C, Ecker JR (2004) Regulation of ethylene gas biosynthesis by the Arabidopsis ETO1 protein. Nature 428:945-950. doi:10.1038/nature02516

Wang Y, Liu C, Li K, Sun F, Hu H, Li X, Zhao Y, Han C, Zhang W, Duan Y, Liu M, Li X (2007) Arabidopsis EIN2 modulates stress response through abscisic acid response pathway. Plant Mol Biol 64:633-644. doi:10.1007/s11103-007-9182-7

Xiong LM, Zhu JK (2003) Regulation of abscisic acid biosynthesis. Plant Physiol 133:29-36. doi:10.1104/pp.103.025395

Xiong L, Ishitani M, Lee H, Zhu JK (2001) The Arabidopsis LOS5/ $A B A 3$ locus encodes a molybdenum cofactor sulfurase and modulates cold and osmotic stress-responsive gene expression. Plant Cell 13:2063-2083. doi:10.1105/tpc.13.9.2063

Xiong LM, Lee H, Ishitani M, Zhu JK (2002) Regulation of osmotic stress-responsive gene expression by the LOS6/ABA1 locus in Arabidopsis. J Biol Chem 277:8588-8596. doi:10.1074/jbc. M109275200

Yanagisawa S, Yoo S-D, Sheen J (2003) Differential regulation of EIN3 stability by glucose and ethylene signalling in plants. Nature 425:521-525. doi:10.1038/nature01984

Zhou L, Jang JC, Jones TL, Sheen J (1998) Glucose and ethylene signal transduction crosstalk revealed by an Arabidopsis glucose-insensitive mutant. Proc Natl Acad Sci USA 95:1029410299. doi:10.1073/pnas.95.17.10294

Zhu S-Y, Yu X-C, Wang X-J, Zhao R, Li Y, Fan R-C, Shang Y, Du SY, Wang X-F, Wu F-Q, Xu Y-H, Zhang X-Y, Zhang D-P (2007) Two calcium-dependent protein kinases, CPK4 and CPK11 regulate abscisic acid signal transduction in Arabidopsis. Plant Cell 19:3019-3036. doi:10.1105/tpc.107.050666 